Article

\title{
Hydrothermal Alteration of Eudialyte-Hosted Critical Metal Deposits: Fluid Source and Implications for Deposit Grade
}

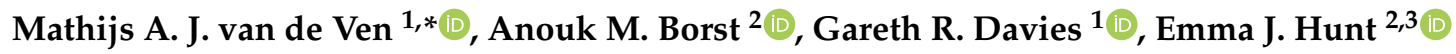 \\ and Adrian A. Finch ${ }^{2}$ \\ 1 Faculty of Earth and Life Sciences, University of Amsterdam, Boelelaan 1085, \\ 1081 HV Amsterdam, The Netherlands \\ 2 School of Earth \& Environmental Sciences, University of St Andrews, North Street, \\ St Andrews KY16 9AL, UK \\ 3 School of Geosciences, University of the Witwatersrand, Private Bag 3, Johannesburg 2050, South Africa \\ * Correspondence: mathijs.vd.ven@outlook.com
}

Received: 30 May 2019; Accepted: 5 July 2019; Published: 10 July 2019

\begin{abstract}
Eudialyte-hosted critical metal deposits potentially represent major sources of rare earth elements (REE), zirconium and niobium. Here, we study the chemical and isotopic composition of fresh and altered eudialyte in nepheline syenite from the Ilímaussaq Complex, Greenland, one of the world's largest known eudialyte-hosted deposits. Late-magmatic hydrothermal alteration caused partial replacement of primary magmatic eudialyte by complex pseudomorph assemblages of secondary $\mathrm{Zr}-, \mathrm{Nb}-$, and REE-minerals. Three secondary assemblage types are characterised by the zirconosilicates catapleiite, gittinsite and zircon, respectively, of which the catapleiite type is most common. To investigate elemental exchange associated with alteration and to constrain the nature of the metasomatic fluids, we compare trace elements and $\mathrm{Sm} / \mathrm{Nd}$ isotope compositions of unaltered eudialyte crystals and their replaced counterparts from five syenite samples (three catapleiite-type, one gittinsite-type, and one zircon-type assemblage). Trace element budgets for the catapleiite-type pseudomorphs indicate a $15-30 \%$ loss of $\mathrm{REE}, \mathrm{Ta}, \mathrm{Nb}, \mathrm{Zr}, \mathrm{Sr}$ and $\mathrm{Y}$ relative to fresh eudialyte. Moreover, the gittinsite- and zircon-type assemblages record preferential heavy REE (HREE) depletion $(\leq 50 \%)$, suggesting that the metasomatic fluids mobilised high field strength elements. Initial Nd isotope ratios of unaltered eudialyte and catapleiite- and gittinsite-type pseudomorphs are indistinguishable, confirming a magmatic fluid origin. However, a higher initial ratio and stronger HREE depletion in the zircon-type pseudomorphs suggests a different source for the zircon-forming fluid. Although alteration reduces the metal budget of the original eudialyte volume, we infer that these elements re-precipitate nearby in the same rock. Alteration, therefore, might have little effect on overall grade but preferentially separates heavy and light REE into different phases. Targeted processing of the alteration products may access individual rare earth families (heavy vs. light) and other metals $(\mathrm{Zr}, \mathrm{Nb}, \mathrm{Ta})$ more effectively than processing the fresh rock.
\end{abstract}

Keywords: eudialyte; hydrothermal alteration; Ilímaussaq; rare earth elements; fluid mobility; peralkaline igneous rocks

\section{Introduction}

With a rare earth element (REE) production that accounted for 70\% of the world's total in 2018 [1], China's dominance in the rare earth market is unrivalled. Strong price fluctuations in 2011 following Chinese export reductions demonstrated the instability of the REE supply chain [2]. Moreover, China is the only country with an integrated REE value chain consisting of hundreds of companies dedicated 
to rare earth research, production, and processing [3]. With applications in clean energy technology, consumer electronics and health care appliances, demand for REE is certain to grow, highlighting the imminent need for the development of new primary resources, as well as advanced processing and recycling chains outside China [3-9].

Eudialyte-hosted critical metal deposits form within peralkaline igneous systems and are increasingly recognised as a potential source of REE and other high field strength elements (HFSE) [10,11]. For example, the Kringlerne deposit in the Mesoproterozoic Ilímaussaq complex, South Greenland, presents a major repository of REE, Zr and $\mathrm{Nb}$ (inferred resource $29 \mathrm{Mt}$ rare earth oxide [12]), hosted by a magmatic floor sequence of layered agpaitic nepheline syenite, locally known as kakortokite [10,13]. Agpaitic nepheline syenite crystallises from highly peralkaline, halogen-rich melts and is identified by the presence of complex Na-Ca-HFSE silicates such as eudialyte- and rinkite-group minerals instead of more common (miaskitic) HFSE-phases like zircon, baddeleyite and titanite [14]. Eudialyte sensu lato, the primary ore mineral at Kringlerne, is an alkali-zirconosilicate with the simplified chemical formula $\mathrm{Na}_{15}(\mathrm{Ca}, \mathrm{REE})_{6}(\mathrm{Fe}, \mathrm{Mn})_{3} \mathrm{Zr}_{3} \mathrm{Si}\left(\mathrm{Si}_{25} \mathrm{O}_{72}\right)\left(\mathrm{O}, \mathrm{OH}, \mathrm{H}_{2} \mathrm{O}\right)_{3}(\mathrm{Cl}, \mathrm{OH})_{2}[15]$ that constitutes up to $40 \%$ of the mode in some kakortokite layers. Eudialyte forms a major host for HFSE with average concentrations in Ilímaussaq of $12 \mathrm{wt} \% \mathrm{ZrO}_{2}, 1 \mathrm{wt} \% \mathrm{Nb}_{2} \mathrm{O}_{5}$ and $1.5-2 \mathrm{wt} \%$ $\mathrm{REE}_{2} \mathrm{O}_{3}$ [16]. Eudialyte has a relatively flat REE pattern, and is substantially more enriched in $\mathrm{Y}$ and heavy REE (HREE) than conventional carbonatite-hosted ore minerals such as bastnäsite, apatite and monazite [17], while having comparatively low concentrations of actinides (U and Th $<100$ ppm [18]). Additionally, eudialyte is easily concentrated by magnetic separation and is highly susceptible to mineral acids, thus presenting a potentially low-cost, low-impact ore mineral [19-21].

Late-stage hydrothermal alteration of eudialyte is inextricably linked to agpaitic complexes and is often attributed to the fluid-rich nature of the melts [22-28]. In Ilímaussaq, eudialyte alteration ranges from localised breakdown along crystal edges and cracks to complete pseudomorphic replacement. The alteration product is a complex intergrowth of microcrystalline zirconosilicates, aluminosilicates and $\mathrm{Nb}$ - and REE-phases [27,29,30]. Two distinct types of pseudomorph assemblages were initially distinguished in Ilímaussaq, characterised by the dominant secondary zirconosilicates catapleiite and zircon [29-31]. The catapleiite-type alteration is widespread and accounts for more than $80 \%$ of breakdown parageneses, whereas the zircon-type has only been described from the most pervasively altered marginal pegmatites that border the intrusion, as well as in a hybrid syenite cross-cutting the kakortokite [29,30,32]. A third alteration assemblage, characterised by gittinsite and superimposed on an earlier catapleiite-bearing paragenesis, was identified by Borst et al. [27]. These authors proposed that a magmatically derived fluid, with relatively high water activity $\left(\alpha \mathrm{H}_{2} \mathrm{O}\right)$, produced the catapleiite-type alteration and a second, Ca-, Sr-bearing magmatic fluid, with high calcium activity $(\alpha \mathrm{CaO})$ and lower alkalinity formed the gittinsite-bearing assemblage. An externally derived fluid has been implicated for the zircon-type alteration [29]. An overview of the composition of the important secondary HFSE-bearing phases for each type of alteration is presented in Table 1.

Borst et al. [27] suggested that eudialyte replacement was mass-conserving for HFSE on the crystal scale, based on qualitative mass-balance studies using mineral compositions and volume, but a quantitative analysis of breakdown assemblages is unavailable. Here, we present a direct comparison of trace element budgets from unaltered eudialyte crystals and their fully replaced counterparts to quantify elemental gains and losses associated with eudialyte alteration. These data allow us to investigate elemental exchange during hydrothermal alteration and provide new insights into the nature and metal-mobilising capacity of the altering fluids. Additionally, we analyse $\mathrm{Nd}$ isotope systematics of primary eudialyte and its replacement products to investigate the origin of the alteration fluids and to understand better the isotopic variation in the magmatic units of the Ilímaussaq complex. Eudialyte-pseudomorph pairs from three rock specimens displaying the catapleiite-type of alteration and one specimen displaying the gittinsite-type of alteration were investigated, as well as zircon-bearing pseudomorphs from a pervasively altered rock sample. Four additional REE-minerals (rinkite-(Ce), nacareniobsite-(Ce), vitusite-(Ce) and epidote) from various levels in the Ilímaussaq 
stratigraphy were analysed to provide a comparison of initial $\mathrm{Nd}$ isotope signatures and to investigate variation in these ratios throughout the Ilímaussaq complex.

Table 1. Important HFSE-bearing phases in the three different eudialyte alteration parageneses.

\begin{tabular}{|c|c|c|c|c|}
\hline Mineral & Formula & $\begin{array}{l}\text { Catapleiite } \\
\text { Type } \\
1-4\end{array}$ & $\begin{array}{l}\text { Zircon } \\
\text { Type }^{1-3}\end{array}$ & $\begin{array}{l}\text { Gittinsite } \\
\text { Type }\end{array}$ \\
\hline Catapleiite & $(\mathrm{Na}, \mathrm{Ca})_{2} \mathrm{ZrSi}_{3} \mathrm{O}_{9} \cdot 2 \mathrm{H}_{2} \mathrm{O}$ & $x$ & $x$ & $x$ \\
\hline Zircon & $\mathrm{ZrSiO}_{4}$ & & $x$ & \\
\hline Gittinsite & $\mathrm{CaZrSi}_{2} \mathrm{O}_{7}$ & & & $x$ \\
\hline Sr-Eudialyte & $\begin{array}{l}(\mathrm{Na}, \mathrm{Sr})_{15} \mathrm{Ca}_{6}(\mathrm{Fe}, \mathrm{Mn})_{3} \mathrm{Zr}_{3}(\mathrm{Si}, \mathrm{Nb})_{2} \\
\mathrm{Si}_{24} \mathrm{O}_{72}\left(\mathrm{O}, \mathrm{OH}, \mathrm{H}_{2} \mathrm{O}\right)_{3}(\mathrm{Cl}, \mathrm{OH})_{2}\end{array}$ & & & $x$ \\
\hline Aegirine & $\mathrm{NaFe}\left(\mathrm{Si}_{2} \mathrm{O}_{6}\right)$ & $x$ & $x$ & $x$ \\
\hline Alkali feldspar & $(\mathrm{Na}, \mathrm{K}) \mathrm{AlSi}_{3} \mathrm{O}_{8}$ & $x$ & $x$ & \\
\hline Analcime & $\mathrm{NaAlSi}_{2} \mathrm{O}_{6} \cdot \mathrm{H}_{2} \mathrm{O}$ & $x$ & $x$ & $x$ \\
\hline Pectolite & $\mathrm{NaCa}_{2} \mathrm{Si}_{3} \mathrm{O}_{8}(\mathrm{OH})$ & $x$ & $\mathrm{x}$ & $x$ \\
\hline Annite & $\mathrm{KFe}_{3}\left(\mathrm{AlSi}_{3} \mathrm{O}_{10}\right)(\mathrm{OH})_{2}$ & & & $x$ \\
\hline Fluorite & $\mathrm{CaF}_{2}$ & $x$ & $x$ & $\mathrm{x}$ \\
\hline Nacareniobsite-(Ce) & $\mathrm{Na}_{3} \mathrm{Ca}_{3}(\mathrm{REE}) \mathrm{Nb}\left(\mathrm{Si}_{2} \mathrm{O}_{7}\right)_{2} \mathrm{OF}_{3}$ & $x$ & $x$ & $\mathrm{x}$ \\
\hline Allanite-(Ce) & $(\mathrm{CaCe})\left(\mathrm{Al}_{2} \mathrm{Fe}\right) \mathrm{Si}_{2} \mathrm{O}_{7}\left(\mathrm{SiO}_{4}\right) \mathrm{OOH}$ & & $x$ & $x$ \\
\hline $\begin{array}{l}\text { A1-type } \\
\text { Ca-REE-P-silicates }{ }^{1,2}\end{array}$ & $(\mathrm{Ca}, \mathrm{Ba}, \mathrm{Ce})_{5}\left(\mathrm{SiO}_{4}, \mathrm{PO}_{4}\right)_{3}(\mathrm{OH}, \mathrm{F})$ & $x$ & $\mathrm{x}$ & $x$ \\
\hline Apatite & $\mathrm{Ca}_{4.5} \mathrm{REE}_{0.5}\left(\mathrm{PO}_{4}\right)_{3}(\mathrm{~F}, \mathrm{Cl}, \mathrm{OH})$ & $x$ & $x$ & \\
\hline Monazite-(Ce) & $(\mathrm{Ce}, \mathrm{La}, \mathrm{Nd}, \mathrm{Th}) \mathrm{PO}_{4}$ & $x$ & $x$ & \\
\hline Fergusonite-(Y) & $\mathrm{YNbO}_{4}$ & & $x$ & $x$ \\
\hline
\end{tabular}

\section{Geological Setting}

The Ilímaussaq complex (1160 $\pm 5 \mathrm{Ma}$ [33]) is one of the youngest intrusions of the Gardar Province, South Greenland. This province is the product of a two-stage rifting event (1300-1250 Ma, 1180-1140 Ma [34]) associated with the break-up of the Columbia Supercontinent. It constitutes dyke swarms, a volcanic-sedimentary graben fill sequence (the Eriksfjord Formation) and about a dozen volcanic igneous centres. Gardar magmas span a compositional range from alkali basalt to trachyte, alkali granite and strongly peralkaline nepheline syenites with local occurrences of lamprophyre and carbonatite [35].

Ilímaussaq hosts some of the most chemically evolved magmatic rocks on Earth and is the type locality for agpaitic rocks. The complex was emplaced at 3 to $4 \mathrm{~km}$ depth $(1 \mathrm{kbar}[36,37])$, intruding into the Eriksfjord Formation and granites of the Julianehåb Batholith (1800 Ma [38]). The complex is roughly disc-shaped with approximately $1500 \mathrm{~m}$ of vertical exposure and horizontal dimensions of about 17 by $8 \mathrm{~km}$. Geophysical studies have shown that the complex does not continue far at depth [37]. Emplacement occurred by at least four successive melt batches that were derived from a common, deep-seated magma chamber [39]. The first batch crystallised a metaluminous augite syenite that is preserved in the roof zone and around the intrusion to the south (Figure 1). The second melt batch produced a thin sheet of peralkaline granite and quartz syenite. The third and fourth melt batches delivered the volumetrically dominant sequences, most of which crystallised eudialyte-group minerals. The third and fourth batch cored the earlier units and formed syenite and nepheline syenite, subdivided into a roof and a floor sequence, separated by a sandwich horizon representing the final and most evolved melt fraction. Crystallisation of the syenite started from the roof and progressed downwards to form conformable layers of pulaskite, foyaite, sodalite foyaite and a poikilitic sodalite-arfvedsonite-eudialyte-nepheline syenite known locally as "naujaite" (cf. [40], for a recent description of these rock types). The lowermost exposed sequence, locally termed "kakortokite", consists of medium- to coarse-grained agpaitic nepheline syenites, most of which are rhythmically layered. The kakortokite postdates the naujaite and is interpreted as a magmatic floor sequence, although the true bottom of the magma chamber is not exposed. The kakortokite 
sequence is subdivided from bottom to top into three structural subunits: the lower layered kakortokite (LLK), slightly layered kakortokite (SLK) and transitional layered kakortokite (TLK) [13]. A marginal pegmatite zone, about 50-100 m wide, separates the kakortokite from the augite syenite [13]. The TLK conformably grades upwards into finer-grained and strongly foliated melanocratic eudialyte-nepheline syenite known as "lujavrite". The lujavrite occurs in aegirine and arfvedsonite dominated varieties, of which the latter represents the chemically most evolved rock type of the complex [41]. The lujavrite and the kakortokite represent the fourth and final melt batch but may have been formed by several pulses of melt [39].



Figure 1. Geological map of the Ilímaussaq complex with sampling locations—from Borst et al. [42] modified after Upton [34]. 
The kakortokite is well-exposed along the Kringlerne coast, south of the Kangerluarsuk fjord (Figure 1). It constitutes a modal mineralogy of alkali feldspar, nepheline, arfvedsonite and eudialyte with minor sodalite, aegirine, aenigmatite and fluorite. The LLK forms an approximately 220-metre-thick sequence consisting of at least 29 tripartite modally-layered units. Each unit is on average $8 \mathrm{~m}$ thick and consists of a basal black layer dominated by arfvedsonite followed by a thin red layer rich in eudialyte (sometimes poorly developed) and sealed by a thick white top layer rich in feldspar and nepheline. Layers are numbered -11 to +17 relative to a well-developed marker horizon 0 and suffixed with a letter indicating their respective colour (B for black, $\mathrm{R}$ for red, W for white) [13]. A fine-grained unlayered melanocratic rock type intersects the LLK between unit -7 and -2 and has most commonly been referred to as a "slumped" kakortokite (after [13]), or more recently described as a "hybrid" sequence between a more primitive Ti-rich melt that mixed with the kakortokite crystal mush [32]. The approximately 50-metre-thick sequence of SLK starts on top of the last recognisable three-layer unit $(+17)$, but, due to poor exposure and severe alteration, a detailed investigation is unavailable to date. The overlying, approximately 60-metre-thick TLK crops out north of the Lakseelv Valley and shows an upward decrease in grain size and an increase in the ratio of aegirine to arfvedsonite [16]. Layering is less pronounced and rhythmic than in the LLK and identified units, separated by eudialyte-rich horizons, were labeled with the letters A to I from top to bottom [13].

\section{Materials and Methods}

\subsection{Sampling Procedure}

Samples were obtained from collections of the authors (prefixes E.J.H. and A.F.), the Geological Survey of Denmark and Greenland, Copenhagen, Denmark (GEUS, sample numbers starting with "109" collected by Bohse and Brooks in 1976, and "520" or "540" collected by author A.B. in 2013)) and N.J. Horsburgh, University of St Andrews, St Andrews, United Kingdom (prefix N.J.H.). Kakortokite samples were selected for geochemical analysis based on the abundance of altered eudialyte, the size of the eudialyte crystals and stratigraphic position. Four pairs of fresh eudialytes and their fully replaced pseudomorphs, and one sample only containing pseudomorphs, from each of the five different kakortokite specimens were prepared for trace element and isotopic analyses. Three specimens originate from the LLK (layer $0,+3$ and +13 ), one from the TLK (level A) and one from the "hybrid" syenite. Additionally, four REE-rich minerals originating from the marginal pegmatite zone (rinkite-(Ce)), from the arfvedsonite-lujavrite (vitusite-(Ce), nacareniobsite-(Ce)) and from the roof zone (epidote) were investigated to provide additional constraints on magmatic initial $\mathrm{Nd}$ isotope compositions. An overview of the samples is given in Table 2.

The kakortokite specimens were cut into slabs and polished to expose mineral surfaces (Figure S1). Pseudomorphs after eudialyte are readily identified under the binocular microscope by their euhedral, equidimensional crystal outline and beige-yellow colour. Pseudomorphs were marked in the section and carefully sampled through micro-milling using the New Wave Research Micro-mill at the Vrije Universiteit Amsterdam following the procedure of Charlier et al. [43] (Figure 2). Sample-water slurries were sequentially removed using a pipette, collected in cleaned glass vials and dried on a hotplate prior to weighing and acid digestion. Fresh eudialyte was handpicked from crushed samples under the binocular microscope, taking care to select only optically clear crystals with clean vitreous surfaces that were unaffected by alteration. Due to insufficient fresh eudialyte in sample 109211, unaltered eudialyte was also collected using the micro-mill. Using image analysis, modal compositions of all rock slabs were determined to model the effects of contamination of the drill-sample by matrix minerals (Table S1, Figure S2). Following digestion in Teflon ${ }^{\mathrm{TM}}$-jacketed aluminum bombs in a mixture of $\mathrm{HCl}-\mathrm{HF}-\mathrm{HNO}_{3}$ and nitration, all samples were dissolved in $2 \mathrm{M} \mathrm{HNO}_{3}$, except the rinkite-(Ce) and nacareniobsite-(Ce) samples, which were dissolved using a mixture of $\mathrm{HNO}_{3}-\mathrm{H}_{2} \mathrm{O}_{2}-\mathrm{HF}$. Total solutions were weighed and aliquots taken for trace element and isotope analysis. Sample preparation and analysis were all performed at the Vrije Universiteit Amsterdam (Amsterdam, The Netherlands). 

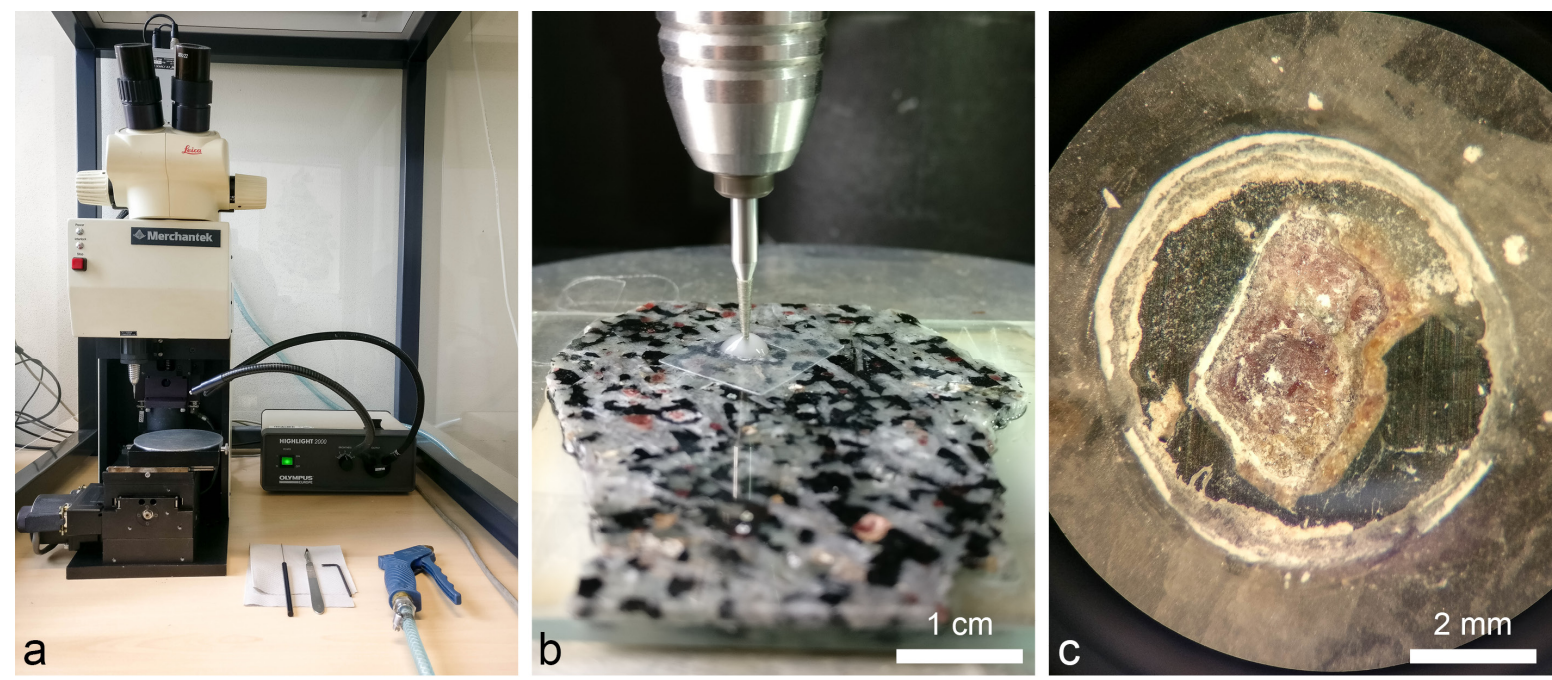

Figure 2. Materials, procedure and result of the drilling procedure. (a) the micro-mill setup in the laminar flowhood; (b) drilling eudialyte from a test sample using a drop of Milli-Q water to capture the milled material; (c) result after the first drilling step.

\subsection{Sample Preparation and Analytical Procedure}

Aliquots for trace element analysis were diluted to a solution of $5 \% \mathrm{HNO}_{3}$ containing at most $30 \mathrm{ng} \mathrm{mL}{ }^{-1} \mathrm{Ce}$, based on typical element concentrations reported in the literature (nacareniobsite-(Ce): [44]; rinkite-(Ce): [45]; vitusite-(Ce): [46]; epidote: [47]; eudialyte (fresh and pseudomorph Ce concentrations assumed to be the same): [16]). Samples were analysed on a Thermo Scientific X-Series II Quadrupole Inductively Coupled Plasma Mass Spectrometer (ICPMS) using a BHVO-2 solution as an external calibration standard. Samples were analysed on 26 July 2017 and a repeat analysis was performed on 8 August 2017. The average of these analyses is presented in results Section 4.

Aliquots for isotopic analysis were spiked with a mixed ${ }^{150} \mathrm{Nd} /{ }^{149} \mathrm{Sm}$ tracer and underwent two column passes-the first employing Eichrom TRU ${ }^{\mathrm{TM}}$ resin for extraction of light rare earth elements and the second using Eichrom $\mathrm{LN}^{\mathrm{TM}}$ resin to separate Sm and Nd. Samples were dried, nitrated and loaded on degassed double Re filaments with no additional activator. Samarium and neodymium isotope ratios were analysed in static mode on a Thermo Scientific Triton Plus Thermal Ionisation Mass Spectrometer (TIMS). Mass fractionation corrections were performed offline using the ${ }^{147} \mathrm{Sm} /{ }^{152} \mathrm{Sm}$ ratio for $\mathrm{Sm}$ and the ${ }^{146} \mathrm{Nd} /{ }^{144} \mathrm{Nd}$ ratio for $\mathrm{Nd}$. Internal precision was monitored by repeated analysis of the JNdi Nd standard [48] and an in-house Sm standard. Both standards performed within the expected range of long-term reproducibility (Tables S2 and S3) and no correction was made.

\section{Results}

\subsection{Petrography}

The primary kakortokite mineralogy consists of arfvedsonite, alkali feldspar, nepheline, eudialyte s.l. and sodalite in varying modal proportions. Alkali feldspar commonly forms coarse laths $(\leq 6 \mathrm{~mm})$ of exsolved or tiled, simple twinned and partially albitised perthite, or as fine-grained $(<0.2 \mathrm{~mm})$ interstitial clusters of albite laths. Arfvedsonite occurs as a euhedral phase in black kakortokite and is interstitial in white and red kakortokite. Eudialyte generally occurs as a euhedral phase (exception: 109211) ranging in size from up to $3 \mathrm{~mm}$ in the LLK to less than $1 \mathrm{~mm}$ in the TLK. Black and white kakortokites commonly show lamination of amphibole and/or feldspar, whereas red kakortokite typically has a saccharoidal texture and less prominent lamination. Minor phases in the kakortokite include aegirine, aenigmatite, rinkite, fluorite and analcime, varying in abundance between units. Fresh and fully replaced eudialyte crystals occur in close spatial association and bear no clear correlation to potential fluid conduits in the rock such as veins and fractures (Figure 3a,b). 
Alteration commonly initiates at the crystal edge or along cracks within the crystal and develops progressively inwards (Figure 3). Alteration minerals identified optically include aegirine, analcime, catapleiite and feldspar (Figure 3a-d) but also annite, allanite, unnamed REE-silicate "A1" [29,30], nacareniobsite-(Ce) and pectolite among others (Figure 3e,f, [27]). The alteration assemblages of the sample set studied here were characterised in previous studies $[27,32]$ and are not further detailed here. The sample major mineralogy is summarised in Table 2 and petrographic descriptions of individual samples are enclosed in Appendix A.

Table 2. Sample overview and major mineralogy of the kakortokite samples.

\begin{tabular}{|c|c|c|c|c|c|c|c|c|c|c|}
\hline $\begin{array}{l}\text { Sample } \\
\text { Code }\end{array}$ & $\begin{array}{l}\text { Stratigraphic } \\
\text { Level }\end{array}$ & $\begin{array}{l}\text { Alteration } \\
\text { Type }\end{array}$ & $\begin{array}{l}\text { Eud } \\
(\%)\end{array}$ & $\begin{array}{l}\text { Pmo } \\
(\%)\end{array}$ & $\begin{array}{l}\text { Fsp } \\
(\%)\end{array}$ & $\begin{array}{l}\text { Nph } \\
(\%)\end{array}$ & $\begin{array}{l}\text { Arf } \\
(\%)\end{array}$ & $\begin{array}{l}\text { Sod } \\
(\%)\end{array}$ & $\begin{array}{l}\text { Anl } \\
(\%)\end{array}$ & $\begin{array}{l}\text { Aeg } \\
\text { (\%) }\end{array}$ \\
\hline \multicolumn{11}{|l|}{ Kakortokite } \\
\hline 109202 & OR & catapleiite & 40 & 30 & 10 & 10 & 5 & 5 & & \\
\hline 109211 & $3 B$ & gittinsite & 2 & 8 & 20 & 20 & 40 & 10 & & \\
\hline 540286 & $13 R$ & catapleiite & 5 & 25 & 30 & 15 & 25 & & & \\
\hline 540269 & TLK-A & catapleiite & 10 & 30 & 10 & 15 & & 10 & 5 & 20 \\
\hline $\mathrm{EJH} / 12 / 091$ & Hybrid & zircon & - & 10 & 60 & 10 & 20 & & & \\
\hline \multicolumn{11}{|l|}{ REE-minerals } \\
\hline $\mathrm{AF} / 16 / 28$ & \multirow{4}{*}{\multicolumn{5}{|c|}{$\begin{array}{l}\text { Roof zone, near Mt Illimaassaq } \\
\text { Black lujavrite } \\
\text { Marginal Pegmatite Kringlerne } \\
\text { Kvanefjeld }\end{array}$}} & \multicolumn{5}{|c|}{ Epidote } \\
\hline $\mathrm{AF} / 16 / 20$ & & & & & & \multicolumn{5}{|c|}{ Vitusite-(Ce) } \\
\hline 520713 & & & & & & \multicolumn{5}{|c|}{ Rinkite-(Ce) } \\
\hline NJH/16/11 & & & & & & \multicolumn{5}{|c|}{ Nacareniobsite-(Ce) } \\
\hline
\end{tabular}

Eud = eudialyte, Pmo = pseudomorph, Fsp = feldspar, Nph = nepheline, Arf = arfvedsonite amphibole, Sod = sodalite, $\mathrm{Anl}=$ analcime and Aeg = aegirine amphibole.

\subsection{Trace Element Data}

Trace element data of fresh eudialyte and micro-milled pseudomorph assemblages are presented in Table 3 and Figures 4-6. The data demonstrate that trace element concentrations and REE patterns for unaltered eudialyte and pseudomorphs are comparable to reported eudialyte compositions from Ilímaussaq. In a chondrite-normalised REE diagram (CI chondrite, [49]), eudialyte and pseudomorph assemblages both display steep LREE patterns and flat HREE patterns, albeit with marginally lower $\mathrm{La} / \mathrm{Ce}$ and higher $\mathrm{Tm} / \mathrm{Yb}$ ratios than reported data. Additionally, all samples display the negative $\mathrm{Eu}$ anomaly characteristic for Ilímaussaq samples [18]. Eudialyte from 540269 (TLK A) is the most LREE-enriched of all samples investigated here and is additionally characterised by comparatively high concentrations of $\mathrm{Rb}$ with respect to other samples (Figure 4). Conversely, REE concentrations in eudialyte from sample 109211 (LLK 0B) are low compared to most reported REE values, whereas the Sr concentration of this sample is relatively high (Figure 4). 

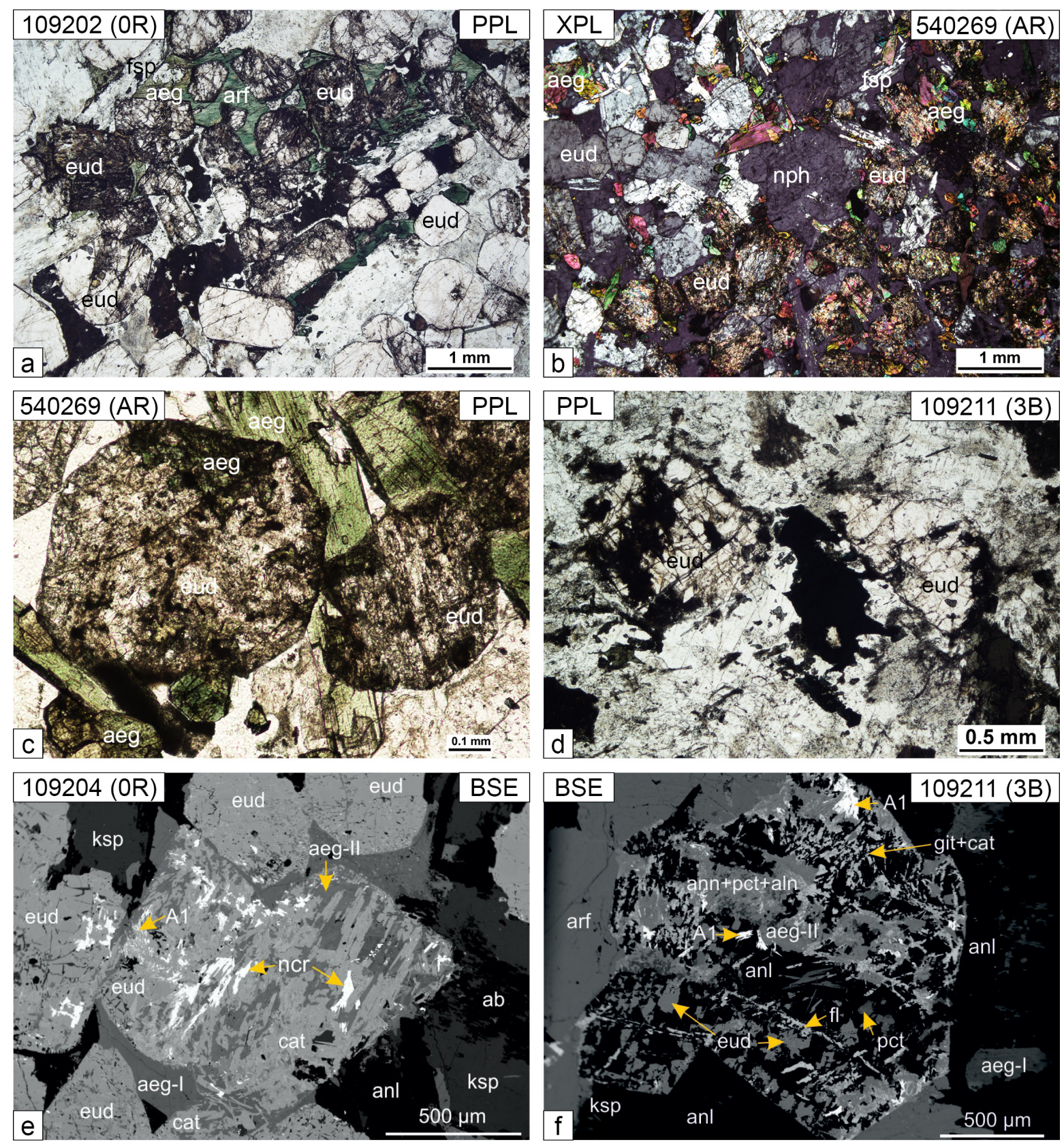

Figure 3. Photomicrographs and back scatter electron (BSE) images of selected samples: (a) altered and fresh octagonal eudialyte under plain polarised light (PPL); (b) altered and unaltered eudialyte under crossed polars (XPL), bright colours due to secondary catapleiite and aegirine and interstitial aegirine; (c) eudialyte pseudomorph with secondary aegirine; (d) partially altered eudialyte crystals; (e) altered eudialyte with secondary catapleiite, nacareniobsite-(Ce), A1, and aegirine; and (f) altered eudialyte with secondary gittinsite. Abbreviations are as follows: A1 = unnamed REE-silicate A1 (see [29]); aeg-I = primary aegirine; aeg-II = secondary aegirine; aln = allanite; anl = analcime; cat = catapleiite; eud = eudialye; $\mathrm{fl}=$ fluorite; git = gittinsite; $\mathrm{ksp}=\mathrm{K}$-feldspar; $\mathrm{ncr}=$ nacareniobsite-(Ce); $\mathrm{nph}=$ nepheline; and pct $=$ pectolite. 
Table 3. Selected major and trace element concentrations as determined by ICPMS (in ppm). Data are averages of two analyses and errors are two relative standard deviations (RSD) of the two analysis series. Concentrations with 2RSD $>30 \%$ are excluded (-), n.d. $=$ not detected. $c p=$ catepleiite-type, gt $=$ gittinsite-type and zr $=$ zircon-type pseudomorph.

\begin{tabular}{|c|c|c|c|c|c|c|c|c|c|c|c|c|c|c|c|c|c|c|c|c|c|c|c|c|c|c|}
\hline \multirow{3}{*}{$\begin{array}{c}\begin{array}{c}\text { ID } \\
\text { Layer } \\
\text { Mineral }\end{array} \\
\text { Li } \\
\text { Be }\end{array}$} & \multicolumn{2}{|c|}{$\begin{array}{c}540286 \quad 2 \text { RSD } \\
+13 \text { Red } \\
\text { Eudialyte } \\
\end{array}$} & \multicolumn{2}{|c|}{$\begin{array}{lc}540286 & \text { 2RSD } \\
\text { +13 Red } \\
\text { Pseudomorph (cp) }\end{array}$} & \multicolumn{2}{|c|}{$\begin{array}{c}540269 \text { 2RSD } \\
\text { TLK-A Red } \\
\text { Eudialyte } \\
\end{array}$} & \multicolumn{2}{|c|}{$\begin{array}{l}540269 \text { 2RSD } \\
\text { TLK-A Red } \\
\text { Pseudomorph (cp) } \\
\end{array}$} & \multicolumn{2}{|c|}{$\begin{array}{c}109202 \quad 2 \text { 2RSD } \\
0 \text { Red } \\
\text { Eudialyte } \\
\end{array}$} & \multicolumn{2}{|c|}{$\begin{array}{l}109202 \quad 2 \text { RSD } \\
\text { 0 Red } \\
\text { Pseudomorph (cp) }\end{array}$} & \multicolumn{2}{|c|}{$\begin{array}{c}\text { EJH/12/091 } 2 \text { RSD } \\
\text { Hybrid } \\
\text { Pseudomorph (zr) } \\
\end{array}$} & \multicolumn{2}{|c|}{$\begin{array}{l}109211 \quad \text { 2RSD } \\
\text { +3 Black } \\
\text { Eudialyte }\end{array}$} & \multicolumn{2}{|c|}{$\begin{array}{l}109211 \quad \text { 2RSD } \\
+3 \text { Black } \\
\text { Pseudomorph (gt) }\end{array}$} & \multicolumn{2}{|c|}{$\begin{array}{c}\text { AF/16/28 } 2 \text { 2RSD } \\
\text { Roof Zone } \\
\text { Epidote }\end{array}$} & \multicolumn{2}{|c|}{$\begin{array}{c}\text { AF/16/20 } 2 \text { RSD } \\
\text { Black Lujavrite } \\
\text { Vitusite-(Ce) }\end{array}$} & \multicolumn{2}{|c|}{$\begin{array}{c}520713 \quad 2 \text { 2RSD } \\
\text { Marg. Pegmatite } \\
\text { Rinkite-(Ce) }\end{array}$} & \multicolumn{2}{|c|}{$\begin{array}{ll}\text { NJH/16/11 2RSD } \\
\text { Kvanefieldt Dumps } \\
\text { Nacareniobsite-(Ce) }\end{array}$} \\
\hline & 6 & & & 0.02 & 22 & 0.04 & & 0.03 & & & & 0.05 & & 0.1 & & & 128 & 0 & & & 602 & & 180 & 0.05 & 165 & 0.25 \\
\hline & 3 & 0.21 & 53 & 0.01 & & & 20 & 0.12 & 13 & 0.14 & 32 & 0.12 & 36 & 0.2 & 9 & 0.27 & & & 2 & 0.19 & 386 & 0.11 & n.d. & & 40 & 0.19 \\
\hline Ti & 837 & 0.12 & 776 & 0.04 & 608 & 0.05 & 457 & 0.19 & & & 761 & 0.06 & & & 589 & 0.02 & - & & 286 & 0.11 & & & 45,957 & 0.18 & 13,311 & 0.15 \\
\hline $\mathrm{Ni}$ & n.d. & & 262 & 0.16 & n.d. & & 275 & 0.26 & n.d. & & 498 & 0.17 & 151 & 0.22 & 71 & 0.25 & & & & & & & n.d. & & n.d. & \\
\hline Ga & 85 & 0.24 & , & 0.08 & 110 & 0.11 & 113 & 0.04 & 85 & $\begin{array}{l}0.22 \\
0.10\end{array}$ & 97 & 0.07 & 122 & 0 & 92 & 0.01 & 97 & 0.13 & 76 & 0.03 & 661 & 0.21 & 685 & $\begin{array}{l}0.12 \\
0.27\end{array}$ & $\therefore$ & \\
\hline $\mathrm{Rb}$ & $\begin{array}{l}25 \\
716\end{array}$ & 0.19 & $\begin{array}{l}342 \\
397\end{array}$ & 0.11 & $\begin{array}{l}86 \\
657\end{array}$ & 0.15 & 128 & 0.18 & 38 & 0.18 & $\begin{array}{l}135 \\
48\end{array}$ & $\begin{array}{l}0.15 \\
0.05\end{array}$ & $\begin{array}{c}96 \\
9619\end{array}$ & 0.16 & $\begin{array}{c}32 \\
3387\end{array}$ & 0.16 & $\begin{array}{l}147 \\
1482\end{array}$ & 0.07 & 2613 & 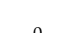 & 1755 & 0.03 & 16 & 0.27 & 388 & 0.21 \\
\hline $\begin{array}{l}\text { Sr } \\
\text { Y }\end{array}$ & $\begin{array}{c}716 \\
5768\end{array}$ & $\begin{array}{c}0 \\
0.08\end{array}$ & $\begin{array}{c}397 \\
4387\end{array}$ & $\begin{array}{l}0.04 \\
0.11\end{array}$ & $\begin{array}{c}657 \\
6257\end{array}$ & $\begin{array}{l}0.07 \\
0.15\end{array}$ & $\begin{array}{l}183 \\
4878\end{array}$ & $\begin{array}{l}0.09 \\
0.16\end{array}$ & $\begin{array}{c}728 \\
5176\end{array}$ & $\begin{array}{l}0.03 \\
0.08\end{array}$ & $\begin{array}{c}478 \\
3886\end{array}$ & $\begin{array}{l}0.05 \\
0.13\end{array}$ & $\begin{array}{l}1612 \\
2847\end{array}$ & $\begin{array}{l}0.01 \\
0.11\end{array}$ & $\begin{array}{l}3187 \\
4551\end{array}$ & $\begin{array}{c}0 \\
0.11\end{array}$ & $\begin{array}{l}1822 \\
2773\end{array}$ & $\begin{array}{l}0.02 \\
0.07\end{array}$ & $\begin{array}{l}2613 \\
2\end{array}$ & $\begin{array}{c}0 \\
0.08\end{array}$ & $\begin{array}{l}4175 \\
3302\end{array}$ & 2.25 & $\begin{array}{c}1837 \\
17.051\end{array}$ & $\begin{array}{l}0.1 \\
0.05\end{array}$ & $\begin{array}{l}5948 \\
4869\end{array}$ & $\begin{array}{l}0.13 \\
0.18\end{array}$ \\
\hline $\mathrm{Zr}$ & $\begin{array}{r}5 / 68 \\
116,361\end{array}$ & $\begin{array}{l}0.08 \\
0.04\end{array}$ & 97,267 & $\begin{array}{l}0.11 \\
0.04\end{array}$ & $\begin{array}{l}6207 \\
99,634\end{array}$ & $\begin{array}{l}.13 \\
0.14\end{array}$ & $\begin{array}{c}4378 \\
73,951\end{array}$ & $\begin{array}{l}0.16 \\
0.16\end{array}$ & $\begin{array}{c}5176 \\
108,994\end{array}$ & $\begin{array}{l}0.08 \\
0.03\end{array}$ & $\begin{array}{r}3886 \\
89,997\end{array}$ & $\begin{array}{l}0.13 \\
0.12\end{array}$ & $\begin{array}{r}29877 \\
59,813\end{array}$ & $\begin{array}{l}0.11 \\
0.09\end{array}$ & $\begin{array}{r}4551 \\
96,756\end{array}$ & $\begin{array}{l}0.11 \\
0.24\end{array}$ & $\begin{array}{r}2773 \\
81561\end{array}$ & $\begin{array}{l}0.07 \\
0.06\end{array}$ & $\begin{array}{l}2 \\
4\end{array}$ & $\begin{array}{l}0.08 \\
0.17\end{array}$ & $\begin{array}{l}3302 \\
1006\end{array}$ & .07 & $\begin{array}{l}17,051 \\
5261\end{array}$ & $\begin{array}{l}0.05 \\
0.02\end{array}$ & $\begin{array}{l}4869 \\
289\end{array}$ & $\begin{array}{l}0.18 \\
0.02\end{array}$ \\
\hline $\mathrm{Nb}$ & 7530 & 0.1 & 5429 & 0.13 & 6038 & 0.17 & 4205 & 0.17 & 8628 & 0.1 & 6474 & $\begin{array}{l}0.12 \\
0.15\end{array}$ & 5057 & 0.11 & $\begin{array}{c}4,700 \\
4782\end{array}$ & $\begin{array}{l}0.24 \\
0.23\end{array}$ & $\begin{array}{l}01,001 \\
3373\end{array}$ & $\begin{array}{l}0.00 \\
0.07\end{array}$ & $\begin{array}{l}4 \\
1\end{array}$ & o.02 & $\begin{array}{l}1000 \\
386\end{array}$ & 0.29 & $\begin{array}{r}3201 \\
43,888\end{array}$ & $\begin{array}{l}0.02 \\
0.05\end{array}$ & 78,965 & $\begin{array}{l}0.02 \\
0.21\end{array}$ \\
\hline Cs & 2 & 0.02 & 3 & 0.01 & 4 & 0.06 & 4 & 0.25 & 3 & 0 & 2 & 0.03 & n.d. & & 6 & 0.12 & 9 & 0.24 & - & & n.d. & & & & & \\
\hline & & & & 0.14 & & & & & & & & & & 0.11 & 753 & & 162 & & 1 & 0.02 & & & nd. & & nd. & \\
\hline La & 4679 & 0.06 & 3617 & 0.09 & 4985 & 0.13 & 3561 & 0.14 & 4529 & 0.07 & 3545 & 0.12 & 3992 & 0.08 & 3418 & 0.09 & 3263 & 0.06 & 2 & 0.08 & 40,307 & 0.01 & 39,311 & 0.05 & 21,917 & 0.19 \\
\hline $\mathrm{Ce}$ & & 0 & 7656 & 0.01 & 10,756 & 0.08 & 78 & 0.13 & 8869 & 0.05 & 7041 & 0.1 & 7254 & 0.06 & 6793 & 0.03 & 5991 & 0.06 & 3 & 0.07 & 99,395 & O. & 99,091 & & & 0.2 \\
\hline Pr & 1022 & 0.06 & 702 & 0 & 1182 & 0.14 & 835 & & 928 & 0.04 & 742 & 0.1 & 14 & 0.1 & 689 & 0.11 & 609 & 0.04 & 1 & 0.11 & 10,658 & 05 & 11,883 & & 9870 & 0.2 \\
\hline $\mathrm{Nd}$ & 3781 & 0.08 & 2862 & 0 & 44 & 0.14 & 3113 & & 3361 & & 2662 & 0. & 198 & 0.08 & 2493 & & 2195 & 0.09 & 2 & 0.13 & & & & & & 0.17 \\
\hline $\mathrm{Sm}$ & 78 & 0. & 5 & & 9 & 0. & 66 & & 675 & & 522 & 0. & 16 & 0.13 & 525 & & 402 & 0.02 & $<1$ & & & & & & & 0.17 \\
\hline Eu & 76 & 0.03 & $\begin{array}{l}53 \\
580\end{array}$ & 0.09 & 90 & 0.16 & 65 & & 66 & & $\begin{array}{l}47 \\
550\end{array}$ & & 402 & & $\begin{array}{l}49 \\
57\end{array}$ & & $\begin{array}{l}34 \\
393\end{array}$ & 0.04 & $<1$ & & & & & & 605 & 0.25 \\
\hline $\mathrm{Gd}$ & 805 & 0.03 & 582 & 0.06 & 926 & 0.12 & 670 & 0.11 & 712 & 0.01 & $\begin{array}{l}530 \\
93\end{array}$ & 0.07 & $\begin{array}{l}402 \\
70\end{array}$ & $\begin{array}{l}0.05 \\
0.09\end{array}$ & $\begin{array}{l}576 \\
108\end{array}$ & 0.06 & 393 & $\begin{array}{l}0.04 \\
0.12\end{array}$ & $<1$ & & $\begin{array}{l}2498 \\
309\end{array}$ & .06 & 103 & $\begin{array}{l}0.05 \\
0.15\end{array}$ & 4041 & 0.2 \\
\hline $\begin{array}{l}\text { Tb } \\
\text { Dy }\end{array}$ & $\begin{array}{l}1111 \\
956\end{array}$ & $\begin{array}{l}0.04 \\
0.04\end{array}$ & $\begin{array}{l}104 \\
715\end{array}$ & $\begin{array}{l}0 . \\
0 .\end{array}$ & $\begin{array}{l}161 \\
1033\end{array}$ & $\begin{array}{l}0.12 \\
0.14\end{array}$ & 7 & & $\begin{array}{l}124 \\
854\end{array}$ & $\begin{array}{l}0.04 \\
0.04\end{array}$ & $\begin{array}{l}93 \\
635\end{array}$ & 0 & 452 & $\begin{array}{l}0.09 \\
0.06\end{array}$ & $\begin{array}{l}108 \\
746\end{array}$ & 0 & $\begin{array}{l}62 \\
430\end{array}$ & $\begin{array}{l}0.12 \\
0.06\end{array}$ & $<1$ & & $\begin{array}{l}309 \\
937\end{array}$ & $\begin{array}{l}0.2 \\
0.27\end{array}$ & $\begin{array}{r}692 \\
3,475\end{array}$ & & $\begin{array}{c}- \\
1537\end{array}$ & 0.19 \\
\hline Ho & 20 & 0. & 15 & & 2 & & 16 & & 18 & & 199 & 0 & & & 168 & & 91 & 0.18 & - & & 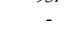 & & & & 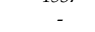 & \\
\hline Er & 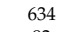 & & 45 & & of & 0. & 49 & & $5 \varepsilon$ & & 438 & & & & 51 & & 303 & 0 & - & & 180 & 0.23 & 1,178 & 0.08 & 195 & .18 \\
\hline $\mathrm{Tm}$ & $\begin{array}{l}92 \\
557\end{array}$ & 0.27 & $\begin{array}{l}73 \\
\end{array}$ & 0.18 & 9 & 0.06 & 72 & & 84 & & 65 & & & & 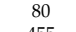 & & 20 & & - & &  & & & & 16 & 0.11 \\
\hline $\mathrm{Yb}$ & $\begin{array}{l}557 \\
66\end{array}$ & $\begin{array}{l}0.02 \\
0.25\end{array}$ & $\begin{array}{l}427 \\
53\end{array}$ & $\begin{array}{l}0.01 \\
0.11\end{array}$ & $\begin{array}{l}548 \\
68\end{array}$ & 0.12 & $\begin{array}{l}423 \\
50\end{array}$ & & $\begin{array}{l}509 \\
57\end{array}$ & 0 & $\begin{array}{l}393 \\
46\end{array}$ & & 293 & & 455 & 0. & 282 & 0.04 & - & & - & & $\begin{array}{l}708 \\
54\end{array}$ & 15 & nd & \\
\hline $\begin{array}{l}\text { Lu } \\
\mathrm{Hf}\end{array}$ & $\begin{array}{r}66 \\
198\end{array}$ & $\begin{array}{l}0 . \\
0 .\end{array}$ & 1653 & $\begin{array}{l}0 \\
0 \\
0\end{array}$ & 1593 & $\begin{array}{l}0 \\
0\end{array}$ & 1180 & $\begin{array}{l}0 \\
0 .\end{array}$ & 2002 & $\begin{array}{l}0.23 \\
0.02\end{array}$ & 1696 & 56 & 51 & $\begin{array}{l}0.12 \\
0.04\end{array}$ & 1663 & $\begin{array}{ll}0 \\
0 \\
0\end{array}$ & 1550 & 0.04 & $<1$ & & 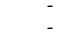 & & $\begin{array}{l}54 \\
105\end{array}$ & 24 & $\begin{array}{l}\text { n.d. } \\
21\end{array}$ & .24 \\
\hline Ta & 447 & 0. & 358 & & 4 & 0 & 291 & 0. & 624 & 0.1 & 489 & & 208 & & 226 & & 294 & 0.18 & & & - & & 968 & 0.09 & 2123 & 0.23 \\
\hline W & 24 & & 27 & & 14 & & 25 & & & & 2 & & 32 & & 592 & & 50 & 0.21 & n.d. & & d. & & n.d. & & n.d. & \\
\hline $\mathrm{Pb}$ & 94 & 0.17 & 54 & 0. & 171 & 0.05 & 27 & & 145 & 0 & 223 & 0. & 68 & & 85 & & 62 & & & & 3864 & .24 & & & & \\
\hline Th & $\begin{array}{l}29 \\
62\end{array}$ & $\begin{array}{l}0 \\
029\end{array}$ & $\begin{array}{l}65 \\
773\end{array}$ & $\begin{array}{l}0.02 \\
0.18\end{array}$ & ${ }_{79}^{62}$ & 0.11 & $\begin{array}{l}28 \\
57\end{array}$ & 0.07 & 24 & $\begin{array}{l}0.02 \\
\end{array}$ & 100 & $\begin{array}{l}0.05 \\
0.9\end{array}$ & 27 & 0.11 & 16 & 0.07 & 27 & 0.02 & - & & 13,934 & 0.16 & 1362 & 0.15 & 317 & 0.21 \\
\hline $\mathrm{La} / \mathrm{Yb}$ & $\begin{array}{l}62 \\
84\end{array}$ & 0.29 & $\begin{array}{l}73 \\
8.5\end{array}$ & 0.18 & $\begin{array}{l}79 \\
91\end{array}$ & & $\begin{array}{l}57 \\
84\end{array}$ & 0.02 & $\begin{array}{l}37 \\
89\end{array}$ & 0.21 & $\begin{array}{l}41 \\
90\end{array}$ & & $\begin{array}{c}20 \\
136\end{array}$ & & $\begin{array}{l}29 \\
75\end{array}$ & 0.03 & 116 & & - & & & & & & & \\
\hline & $\begin{array}{l}8.4 \\
1.4\end{array}$ & & 1. & & $\begin{array}{l}9.1 \\
1.7\end{array}$ & & 1. & & $\begin{array}{l}8.9 \\
1.4\end{array}$ & & & & & & $\begin{array}{l}7.5 \\
1.3\end{array}$ & & 1 & & $\therefore$ & & & & & & & \\
\hline $\mathrm{Sm} / \mathrm{Na}$ & 0.21 & & 0.20 & & 0.21 & & 0.21 & & 0.20 & & 0.20 & & 0.17 & & 0.21 & & 0.18 & & - & & 0.13 & & 0.16 & & 0.17 & \\
\hline
\end{tabular}





Figure 4. (a) chondrite-normalised rare earth element concentrations for eudialyte and pseudomorphs plotted against eudialyte data from Borst et al. [16] (Electron Microprobe, one analysis excluded) and Pfaff et al. [50] (Laser Ablation ICPMS). Concentrations measured from eudialyte cores by Borst et al. are plotted separately—normalisation against CI Chondrite [49]; (b) selected major and trace elements as determined by ICPMS, plotted against literature data for eudialyte; sources as for (a). $\mathrm{cp}=$ catapleiite-type, $\mathrm{gt}=$ gittinsite-type, $\mathrm{zr}=$ zircon-type alteration assemblage.

Figure 4a illustrates that REE concentrations of the pseudomorph samples, although within the range of literature values, are consistently lower than those of fresh eudialyte from the same rock. The similarity between normalised REE patterns of eudialyte and pseudomorphs in Figure 4a suggests that alteration was not associated with strong REE fractionation. For the catapleiite samples, this is illustrated by the observation that eudialyte and pseudomorphs display similar $\mathrm{La} / \mathrm{Yb}$ and $\mathrm{Gd} / \mathrm{Yb}$ ratios (Figure 5). However, high La/Yb ratios in the gittinsite and zircon-type pseudomorph assemblages indicate that alteration is associated with preferential heavy REE depletion (Figure 5). Relative differences in trace element budgets between fresh and altered eudialyte are presented in Figure 6. The figure shows eudialyte-normalised concentration differences and illustrates that REE concentrations in the catapleiite-type pseudomorph are 20-30\% lower than those in eudialyte from the same rock. For the gittinsite-type sample (109211), the loss in REE ranges from $<5 \%$ for La through $>46 \%$ for Ho to $38 \%$ for $\mathrm{Yb}$. Sample EJH/12/091, containing the zircon-bearing eudialyte pseudomorphs, contained no fresh eudialyte, hence the zircon-type pseudomorphs were compared to the average eudialyte composition from this study ( $n=3,109211$ excluded). This yields a REE loss for the zircon-type pseudomorphs of $17 \%$ for La ranging through $>48 \%$ for Sm-Er to $45 \%$ for $\mathrm{Yb}$. 


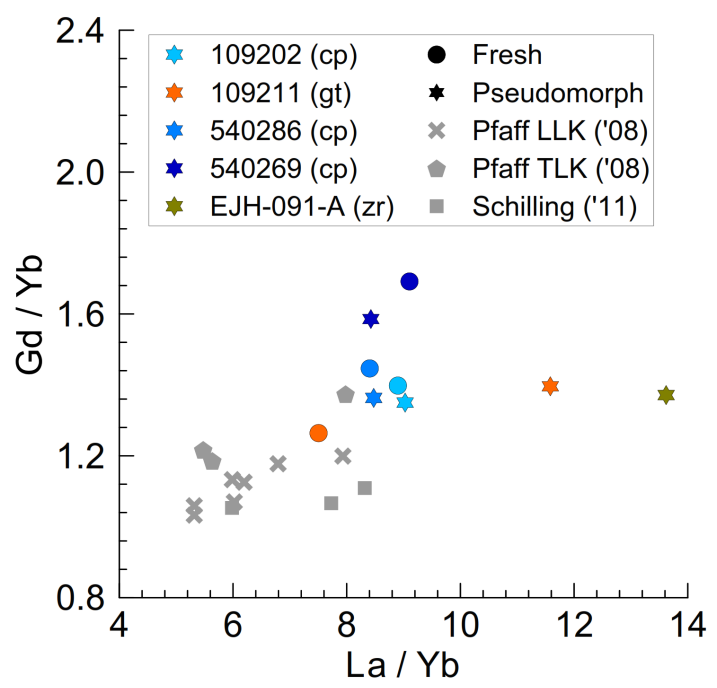

Figure 5. Rare earth element ratios of eudialyte and their pseudomorphs plotted against Laser Ablation ICPMS data for eudialyte from Pfaff et al. [50] and Schilling et al. [18].

In addition to being depleted in REE, the eudialyte pseudomorphs are characterised by lower concentrations of $\mathrm{Pb}, \mathrm{Ba}, \mathrm{Ta}, \mathrm{Nb}, \mathrm{Zr}, \mathrm{Ti}, \mathrm{Y}$ and $\mathrm{Sr}$ and have elevated concentrations of $\mathrm{Rb}, \mathrm{U}$ and Th compared to precursor eudialyte (Figure 6). For the catapleiite-type pseudomorphs, losses in Ta, $\mathrm{Nb}$ and $\mathrm{Y}(20-30 \%)$ are comparable to those of the REE, whereas losses of $\mathrm{Zr}$ (15-25\%) are marginally smaller and losses of $\mathrm{Sr}(34-72 \%)$ and $\mathrm{Ba}(43-75 \%)$ are larger. Addition of $\mathrm{Rb}$ is displayed by all catapleiite-type pseudomorphs with gains ranging from $50 \%$ to $>1200 \%$ (109202). Trends of other elements are less equivocal, with two out of three samples showing loss of $\mathrm{Pb}(40-80 \%)$ and addition of $\mathrm{U}\left(10-18 \%, \mathrm{U}_{\mathrm{pmo}}=20-73 \mathrm{ppm}\right)$ and $\mathrm{Th}(125-300 \%$, Th $\mathrm{pmo}=27-100 \mathrm{ppm})$. Like the catapleiite-type pseudomorphs, the gittinsite-type pseudomorphs lost part of their $\mathrm{Nb}(-29 \%), \mathrm{Y}(-39 \%), \mathrm{Zr}(-15 \%)$, Sr $(-43 \%)$ and $\mathrm{Ba}(-78 \%)$, while $\mathrm{U}(+15 \%), \mathrm{Rb}(+360 \%)$ and $\mathrm{Th}(+74 \%)$ were added. The Ta gain shown in Figure 6 for sample 109211 reflects the low Ta contents in fresh eudialyte from this sample rather than a high Ta content in the pseudomorphs. The zircon-type pseudomorphs have high Ba and Sr compared to average fresh eudialyte (Figure $4 \mathrm{~b}$ ) and these elements appear as apparent gains in Figure 6. Trends for other elements are consistent with the catapleiite-type pseudomorphs.

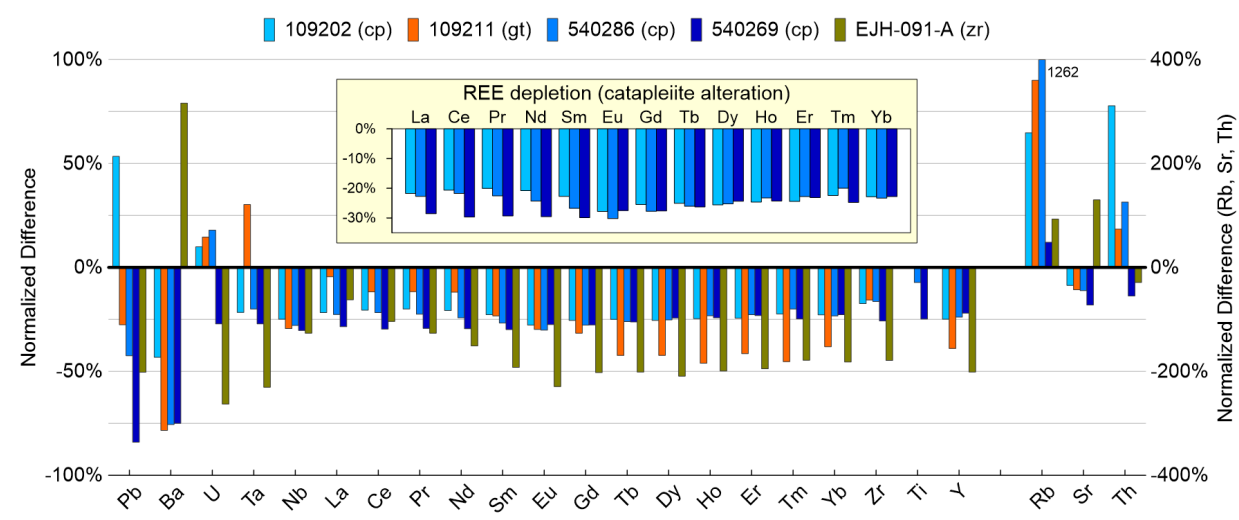

Figure 6. Relative differences in trace element concentrations between pseudomorphs and eudialyte, calculated for an element $X$ as: $\left(\left[X_{\text {pmo }}\right]-\left[X_{\text {eud }}\right]\right) /\left[X_{\text {eud }}\right] \times 100 \%$. Sample EJH/12/091 contains no fresh eudialyte for comparison and was compared to the average eudialyte concentration from this study $(n=3,109211$ excluded from average). Note the different scale for $\mathrm{Rb}$, Sr and Th. 


\subsection{Sm-Nd Isotope Data}

Results of TIMS isotopic analysis are summarised in Table 4 and displayed in Figures 7 and 8. The $\mathrm{Sm} / \mathrm{Nd}$ ratios determined by isotope dilution are consistent with ICPMS results and range from 0.201 to 0.214 for fresh eudialyte and from 0.174 to 0.213 for the pseudomorphs (Figure 7a). Initial $\mathrm{Nd}$ isotope ratios $\left({ }^{143} \mathrm{Nd} /{ }^{144} \mathrm{Nd}_{i}\right)$, calculated for an intrusion age of $1160 \mathrm{Ma}$ [33], range from $0.511083 \pm 8$ to $0.511094 \pm 8$ for eudialyte $(\varepsilon \mathrm{Nd}(\mathrm{t})=-0.9$ to -1.1$)$ and from $0.511087 \pm 7$ to $0.511102 \pm 7$ for the catapleiite- and gittinsite-type pseudomorphs $(\varepsilon \mathrm{Nd}(\mathrm{t})=-0.8$ to -1.0$)$. The zircon-bearing pseudomorph $(\mathrm{EJH} / 12 / 091)$ yields a higher value, outside analytical error, of $0.511166 \pm 7(\varepsilon \mathrm{Nd}(\mathrm{t})=0.5)$. Initial $\mathrm{Nd}$ isotope ratios of other rare earth phases range from $0.511095 \pm 7$ to $0.511098 \pm 5(\varepsilon \mathrm{Nd}(\mathrm{t})=-0.8$ to -0.9$)$ except for the epidote sample, which yields an initial ratio of $0.511055 \pm 11(\varepsilon \mathrm{Nd}(\mathrm{t})=-1.7)$. From Figure $7 \mathrm{~b}$, it appears that the pseudomorphs are characterised by higher initial Nd ratios. However, a Welch's $t$-test for small samples with unequal variance demonstrates that this difference is not significant at the $95 \%$ confidence limit (internal precision not taken into account, Table S4). Except for EJH/12/091, initial Nd ratios reported in the present study are consistent with those reported by Marks et al. [51] for the Ilímaussaq intrusion as a whole and by Borst et al. [42] for kakortokite unit 0 (Figure 7b).

An isochron regression calculated for just pseudomorph data (excluding EJH/12/091) yields $1139 \pm 240 \mathrm{Ma}$ and an initial ratio of $0.51111 \pm 20$ with a mean square weighted deviation (MSWD) of 2.7. The isochron of all datapoints combined, excluding the zircon-pseudomorph and the epidote yields $1136 \pm 32 \mathrm{Ma}$ with an initial Nd ratio of $0.51111 \pm 2(\mathrm{MSWD}=2.4)$ (Figure 8) and a geological scatter in initial $\mathrm{Nd}$ ratios of $0.000008(2 \sigma)$, which is the same order of magnitude as our internal precision. Finally, an isochron of all primary magmatic minerals (i.e., fresh eudialyte and the rare earth phases excluding epidote) yields a similar age of $1133 \pm 18$ with an initial $\mathrm{Nd}$ ratio of $0.51111 \pm 1$ (MSWD = 1.3). 
Table 4. Isotope data of eudialyte, eudialyte pseudomorphs and rare earth minerals. Initial ratios calculated for an intrusion age of 1160 Ma using a decay constant for ${ }^{147} \mathrm{Sm}$ of $6.54 \times 1012$ [52] and a relative standard deviation on $\mathrm{Sm} / \mathrm{Nd}$ of 0.005 that was estimated based on repeated measurements of geological standards. Calculation of $\varepsilon \mathrm{Nd}(\mathrm{t})$ and $\mathrm{T}_{\mathrm{CHUR}}$ using a present day CHUR ${ }^{143} \mathrm{Nd} /{ }^{144} \mathrm{Nd}$ ratio of 0.512638 [53] and a ${ }^{147} \mathrm{Sm} /{ }^{144} \mathrm{Nd}$ of 0.1966 [54]. Decay constant and age of intrusion were assumed to be free of error in the calculation of the error on $\varepsilon N d(t)$. Ep $=$ epidote, Vit $=$ vitusite- $(\mathrm{Ce}), \mathrm{Rkt}=$ rinkte-(Ce) and $\mathrm{Ncr}=\mathrm{nacareniobsite}-(\mathrm{Ce})$.

\begin{tabular}{|c|c|c|c|c|c|c|c|c|c|c|c|c|}
\hline ID & Type & Unit & $\frac{S m}{N d}$ & $\frac{{ }^{143} \mathrm{Nd}}{{ }^{144} \mathrm{Nd}}$ & $2 \sigma^{*}$ & $\frac{{ }^{147} S m}{{ }^{144} N d}$ & $2 \sigma^{*}$ & $\frac{{ }^{143} N d}{{ }^{144} N d_{i}}$ & $2 \sigma^{*}$ & $\varepsilon N d(t)$ & $2 \sigma^{*}$ & $T_{C H U R}$ \\
\hline 540286 & Eud & $+13 \mathrm{R}$ & 0.211 & 0.512063 & 0.000005 & 0.1278 & 0.0006 & 0.51109 & 0.000007 & -1.0 & 0.1 & 1.27 \\
\hline 540269 & Eud & TLK-A & 0.215 & 0.512085 & 0.000006 & 0.1302 & 0.0007 & 0.511094 & 0.000008 & -0.9 & 0.2 & 1.24 \\
\hline 109202 & Eud & OR & 0.205 & 0.512037 & 0.000005 & 0.1239 & 0.0006 & 0.511093 & 0.000007 & -0.9 & 0.1 & 1.27 \\
\hline 109211 & Eud & $+3 \mathrm{~B}$ & 0.212 & 0.512058 & 0.000006 & 0.1281 & 0.0006 & 0.511083 & 0.000008 & -1.1 & 0.1 & 1.28 \\
\hline 540286 & Pmo (cp) & $+13 \mathrm{R}$ & 0.203 & 0.512039 & 0.000005 & 0.1230 & 0.0006 & 0.511102 & 0.000007 & -0.8 & 0.1 & 1.26 \\
\hline 540269 & Pmo (cp) & TLK-A & 0.215 & 0.512075 & 0.000005 & 0.1297 & 0.0006 & 0.511087 & 0.000007 & -1.0 & 0.1 & 1.24 \\
\hline 109202 & Pmo (cp) & OR & 0.197 & 0.512006 & 0.000005 & 0.1193 & 0.0006 & 0.511098 & 0.000007 & -0.8 & 0.1 & 1.29 \\
\hline 109211 & Pmo (gt) & $+3 \mathrm{~B}$ & 0.189 & 0.511970 & 0.000005 & 0.1143 & 0.0006 & 0.511100 & 0.000007 & -0.8 & 0.1 & 1.24 \\
\hline $\mathrm{EJH} / 12 / 091$ & Pmo (zr) & Hybrid & 0.174 & 0.511965 & 0.000005 & 0.1049 & 0.0005 & 0.511166 & 0.000007 & 0.5 & 0.1 & 1.12 \\
\hline $\mathrm{AF} / 16 / 28$ & Ep & & 0.182 & 0.511891 & 0.000010 & 0.1097 & 0.0005 & 0.511055 & 0.000011 & -1.7 & 0.2 & 1.31 \\
\hline $\mathrm{AF} / 16 / 20$ & Vit & & 0.126 & 0.511678 & 0.000004 & 0.0761 & 0.0004 & 0.511098 & 0.000005 & -0.8 & 0.1 & 1.21 \\
\hline 520713 & Rkt & & 0.158 & 0.511826 & 0.000005 & 0.0955 & 0.0005 & 0.511098 & 0.000006 & -0.8 & 0.1 & 1.22 \\
\hline NJH/16/11 & Ncr & & 0.173 & 0.511891 & 0.000005 & 0.1045 & 0.0005 & 0.511095 & 0.000007 & -0.9 & 0.1 & 1.24 \\
\hline
\end{tabular}

* double standard error. 



Figure 7. (a) Sm/Nd ratios for all samples calculated from TIMS data and compared to ICPMS data; (b) plot of initial $\mathrm{Nd}$ isotope ratios of fresh eudialyte, their pseudomorphs and other rare-earth-bearing minerals. Error bars are propagated double standard errors $(2 \sigma)$ on initial $\mathrm{Nd}$ ratios. Most data from the present study occupy a narrow range (dashed lines, excluding two outliers, see discussion). Shown for comparison are initial $\mathrm{Nd}$ ratios reported by Borst et al. [42] for whole rocks and mineral separates from kakortokite unit 0 (TIMS + ICPMS, dark grey box) and by Marks et al. [51] for mineral separates and one whole rock from a range of Ilímaussaq samples including an augite syenite, lujavrite and a naujaite (TIMS, light grey box). HKK = hybrid kakortokite, mineral abbreviations as in Table 4 . 




Figure 8. Sm/Nd isochron calculated with omission of the zircon-bearing pseudomorph (Zr-pmo) and the epidote sample (Ep). Double standard error bars are displayed for both ratios but are smaller than the data markers. The isochron for an intrusion age of $1160 \mathrm{Ma}\left({ }^{143} \mathrm{Nd} /{ }^{144} \mathrm{Nd}_{i}=0.51111\right.$, [33] $)$ is shown for comparison.

\section{Discussion}

\subsection{Eudialyte Alteration and Mobilisation of HFSE}

Mineralogical studies of the peralkaline Ilímaussaq complex identified three distinct eudialyte alteration parageneses, characterised by secondary catapleiite, gittinsite and zircon, respectively $[27,29,30]$. Based on mineralogical and volumetric mass-balance estimates, these authors all concluded that catapleiite-type alteration required an addition of $\mathrm{F}, \mathrm{H}_{2} \mathrm{O}$ and $\mathrm{Al}$ with occasionally $\mathrm{P}$ and $\mathrm{Ba}$, and was associated with loss of $\mathrm{Na}, \mathrm{Si}$ and $\mathrm{Cl}$. Of these elements, only $\mathrm{Ba}$ was included in the current study and was found to be depleted in the pseudomorphs relative to fresh eudialyte (Table 3). In addition, previous studies concluded that the HFSE were largely immobile during eudialyte breakdown based on relative volumetric proportions of secondary REE-, $\mathrm{Nb}$ - and $\mathrm{Zr}$-bearing phases and the fact that secondary phases were generally not observed outside the pseudomorph volumes $[27,29-31,55]$. Our trace element data, however, suggest that each type of sub-solidus eudialyte alteration is associated with elemental mobilisation. In particular, we find that REE, Ta, Nb, Zr, Ba, Y, $\mathrm{Ti}$ and $\mathrm{Sr}$ are lost from the eudialyte pseudomorphs.

To validate these results, we consider two types of sampling bias that could lead to underestimation of pseudomorph HFSE budgets: (1) accidental sampling of HFSE-poor matrix minerals ("dilution") and (2) inability to sample a representative pseudomorph volume due to spatial clustering of secondary REE- and Nb-phases near the pseudomorph margin [55] ("undersampling"). These potential sources of errors were recognised before drilling and hence the greatest care was taken to sample the complete pseudomorph domain without crossing the pseudomorph boundaries. Pseudomorphs were selected based on their well-defined "crystal" boundaries and visual control during the drilling procedure was good, enabling us to sample only the alteration assemblage. Moreover, at least nine pseudomorph domains were sampled from each rock specimen to ensure that a representative pseudomorph sample was collected. Hypothetically, dilution of HFSE would be proportional to the amount of HFSE-poor matrix minerals (nepheline, feldspar, arfvedsonite and sodalite) accidentally sampled so the measured depletions (20-30\%) require addition of up to $30 \%$ matrix material (Figures S3 and S4), which is inconsistent with our careful laboratory practice. Moreover, considering the different modal compositions of each sample, addition of up to $70 \%$ matrix minerals is required to explain the perceived trace element budgets (Figure S4). Given the control 
on the drilling procedure, this is considered unrealistic. Undersampling is arguably harder to test during the drilling procedure as secondary minerals are microscopic in size, but is inconsistent with the coupled depletion of REE, $\mathrm{Nb}$ and $\mathrm{Zr}$. Zirconium is hosted in the pseudomorphs primarily by catapleiite, which is typically evenly distributed across the pseudomorphic intergrowths and would not be affected by undersampling of REE-Nb phases. Additionally, the consistent pattern of 20-30\% REE depletion for all catapleiite pseudomorphs is hard to reconcile with a random sampling bias, which would produce more variable data. For these reasons, we rule out sampling bias as an explanation for the comparatively low HFSE contents of the pseudomorphs.

\subsection{Nature of the Altering Fluids}

Replacement of eudialyte by catapleiite-bearing assemblages has been observed in many eudialyte-bearing complexes and is considered to result from alteration by late-magmatic Na-rich aqueous fluids [23,24,29,56]. Borst et al. [27], based on chemographic modelling, interpreted the gittinsite-type assemblages to be the product of low-temperature sub-solidus alteration by a Ca- and Sr-rich, lower alkalinity aqueous fluid. Additionally, it was suggested that the gittinsite-forming fluid represented a more evolved fluid than the fluid producing the catapleiite-bearing paragenesis, based on textural evidence that gittinsite overprints catapleiite. The zircon-type alteration was inferred to be the product of hydrothermal fluids intruding "along fractures" and originating from late lujavrite melts or from outside the intrusion [29]. Indirect evidence for an external fluid source is provided by the observation that the zircon-type of alteration is most common in the marginal pegmatites, which are restricted to the outer zone of the intrusion. The initial $\mathrm{Nd}$ isotope ratios for pseudomorphs and fresh eudialyte are statistically indistinguishable at the 95\% confidence limit (Table S4) and support a local, magmatic origin for the catapleiite- and gittinsite-forming fluids. Conversely, the positive $\varepsilon \mathrm{Nd}(\mathrm{t})$ value (Figure $7 b$ ) and off-isochron position of the zircon-bearing pseudomorphs (Figure 8) suggest isotopic disturbance occurred and are consistent with an external origin for the alteration fluid. Although we do not have additional $\mathrm{Nd}$ isotopic data for the hybrid kakortokite, positive $\varepsilon N d(t)$ values $(>1)$ have not previously been recorded for any of the Ilímaussaq intrusive units [51] and have only been reported for overlying basalts of the Eriksfjord Formation [57] and a single micro-kakortokite dyke [58]. We thus infer that the positive $\varepsilon \mathrm{Nd}(\mathrm{t})$ value of the zircon-type pseudomorphs reflects modification of the $\mathrm{Nd}$ isotope system through external fluid interaction.

While traditionally considered to be immobile in hydrothermal environments, HFSE undergo substantial late-magmatic mobilisation in alkaline magmatic systems by late-stage fluids rich in ligands such as $\mathrm{Cl}^{-}, \mathrm{F}, \mathrm{PO}_{4}{ }^{3-}, \mathrm{SO}_{4}{ }^{2-}$ and $\mathrm{OH}^{-}$[56,59-65]. Of these ligands $\mathrm{Cl}^{-}, \mathrm{F}^{-}$and $\mathrm{OH}^{-}$and potentially $\mathrm{PO}_{4}{ }^{3-}$ are considered most important in the late-stage fluids at Ilímaussaq, as estimates of redox state suggest that the fluids at Ilímaussaq contained no sulfate ([66], under review). In many alkaline hydrothermal systems, $\mathrm{F}^{-}$is considered the primary REE-transporting ligand, in line with Pearson's rule that hard cations (such as the REE) should bind preferentially to hard anions (such as $\mathrm{F}^{-}$) [67]. However, recent experimental and numerical studies have demonstrated that at relevant hydrothermal temperatures $\left(200-400^{\circ} \mathrm{C}\right), \mathrm{Cl}^{-}$and $\mathrm{SO}_{4}{ }^{2-}$, rather than $\mathrm{F}^{-}$, form the most stable complexes with REE in (oxidised) aqueous solutions [68-70]. These studies interpret that the common association of REE and fluorite in nature is the result of the fluoride ion acting as a binding ligand that promotes REE deposition at neutral to high $\mathrm{pH}$. By contrast, transport of $\mathrm{Nb}$ and $\mathrm{Zr}$ is promoted by the availability of $\mathrm{F}$ in the fluid, although complexation occurs with various hydroxyfluoride species rather than the fluoride ion [71,72]. Markl and Baumgartner [73] emphasised the neutral to basic nature of late-magmatic hydrothermal fluids in Ilímaussaq. Based on this, Borst et al. [27] attributed the inefficiency of HFSE-complexation and hydrothermal mobilisation of HFSE during eudialyte breakdown to the insolubility of REE-fluoride phases at neutral to high $\mathrm{pH}$. Furthermore, they hypothesised that the F-bearing aqueous fluids would immediately react with the Ca and REE released from eudialyte to precipitate fluorite and F-bearing REE-minerals (e.g., A1 and nacareniobsite-(Ce)). The associated removal of F from the fluid would furthermore destabilise $\mathrm{Nb}$ - and $\mathrm{Zr}$-binding hydroxyfluoride compounds [71,72] and 
cause the deposition of these elements into nacareniobsite-(Ce) and catapleiite [27]. The present study, however, suggests that eudialyte alteration in the kakortokite is associated with substantial transport of $\mathrm{REE}, \mathrm{Zr}$ and $\mathrm{Nb}$ from the eudialyte pseudomorphs. From this, we could infer that the late-magmatic fluids at work here were either not as basic as those described by Markl and Baumgartner [73], or that HFSE are more easily mobilised by $\mathrm{Cl}^{-}, \mathrm{OH}^{-}$and $\mathrm{F}^{-}$complexation than previously assumed under these conditions.

\subsection{Nd Isotopes: Evidence for Closed-System Fractionation}

The narrow range of $\varepsilon \mathrm{Nd}(\mathrm{t})(-0.8$ to -1.1$)$ observed for most of our samples is comparable to those reported previously for Ilímaussaq (Figure $7 \mathrm{~b}$ ). Borst et al. [42] found a range of -0.4 to -1.1 for mineral separates and whole rocks from kakortokite unit 0, whereas Marks et al. [51] reported a range of -0.1 to -1.8 for a series of amphiboles and whole rock data from various agpaites and two augite syenites. Such weakly negative $\varepsilon \mathrm{Nd}(\mathrm{t})$ values have been interpreted by various authors as reflecting low-degree contamination of mantle-derived magmas with Archean or Proterozoic (Ketilidian) crustal material [42,51,74,75]. The interpretation by Marks et al. [51] that their Nd data were indicative of closed-system fractionation for Ilímaussaq is supported by the present work. Moreover, our data support those authors' interpretation that melts were derived from a mantle source with an $\varepsilon N d(t)$ of about -1 to -2 and suggest that the mantle source $\varepsilon N d(t)$ was closer to -1 . As such, the narrow range of $\varepsilon \mathrm{Nd}(\mathrm{t})$ values for the agpaitic units of the Ilímaussaq complex is consistent with closed-system fractionation of a mantle melt that experienced minimal amounts of crustal contamination. The epidote sample $(\varepsilon N d(t)=-1.7)$ and the zircon bearing pseudomorphs $(\varepsilon N d(t)=0.5)$ are outliers to this trend. As noted above, we infer the positive $\mathrm{Nd}$ value for the zircon-bearing pseudomorphs to be the result of isotopic disturbance by hydrothermal alteration. The $\varepsilon N d(t)$ value of the epidote sample is within the range reported by Marks et al. [51] but is different outside analytical error from of all kakortokite samples reported here (Figure $7 \mathrm{~b}$ ). Additionally, the epidote is unique among our samples in having a positive $\mathrm{Eu}$ anomaly. This sample was collected from the roof zone, near the contact with the Eriksfjord Formation basalts, which have previously been found to yield negative $\varepsilon N d(t)$ values down to -3.2 [76]. We suggest that the positive Eu anomaly, low $\varepsilon N d(t)$ value and off-isochron position of the epidote sample reflect crustal contamination at the pluton margin.

\subsection{Implications for Resource Potential}

Extractability of the raw commodity is a key factor in defining the economic viability of a mineral occurrence. One of the key determinants of extractability is the mineralogy of the ore, which makes eudialyte-hosted critical metal deposits particularly attractive: eudialyte is easily concentrated by magnetic separation and readily soluble in mineral acids [19-21]. However, hydrothermal alteration of eudialyte commonly produces a complex secondary mineralogy and the present work shows that at Ilímaussaq up to $20-30 \%$ of REE, Nb and Zr may be lost from the primary crystal volume. While it may be that most of these elements re-precipitated within the rock volume, the diverse nature of the secondary mineralogy may complicate the metallurgical process. Although eudialyte is easily dissolved in dilute nitric acid, at least one of the secondary phases, nacareniobsite-(Ce) requires additional treatment with $\mathrm{HF}$ (Section 3.2). Moreover, the grain size reduction associated with eudialyte breakdown - from $\mathrm{mm}$ to $\mu \mathrm{m}$-and changes in the magnetic properties potentially affect the physical concentration of the ore minerals, although no effect on concentrate yields has been reported for Ilímaussaq [77] . To optimise critical metal recovery from eudialyte-hosted deposits, it is important to investigate how the secondary REE-, Nb- and $\mathrm{Zr}$-bearing phases can be incorporated in the ore beneficiation process. If the secondary minerals can be targeted individually, different metals and even REE families (heavy versus light REE) could be liberated in sequence, potentially simplifying the REE separation process. We suggest that a coupling of detailed mineralogical characterisation with physical and metallurgical processing testing is needed to evaluate the limitations and opportunities of the extraction of critical metals from eudialyte alteration assemblages. 


\section{Conclusions}

Eudialyte-hosted critical metal deposits could be an important future source of rare earth elements and other HFSE. However, sub-solidus breakdown of eudialyte into intimate micrometre-scale aggregates of secondary zirconosilicates, aluminosilicates and $\mathrm{Nb}$ - and REE-phases is commonly observed. Our comparison of trace element concentrations of eudialyte and pseudomorph material from the same rocks demonstrates that eudialyte alteration was associated with loss of HFSE from the eudialyte crystal domain. The catapleiite-bearing paragenesis, accounting for over $80 \%$ of alteration parageneses in Ilímaussaq, is associated with up to $20 \%$ loss of $\mathrm{Zr}$ and up to $20-30 \%$ loss of Ta, $\mathrm{Nb}, \mathrm{Y}$ and all REE. This alteration type is further characterised by loss of $\mathrm{Sr}(\leq 20 \%), \mathrm{Ba}$ and $\mathrm{Pb}$ $(\leq 75 \%)$ and enrichment in Th $\left(\mathrm{Th}_{\mathrm{pmo}}=27-100 \mathrm{ppm}\right), \mathrm{U}\left(\mathrm{U}_{\mathrm{pmo}}=20-73 \mathrm{ppm}\right)$ and $\mathrm{Rb}$ but relatively minor REE fractionation. By contrast, high $\mathrm{La} / \mathrm{Yb}$ ratios in the gittinsite- and zircon-type alteration assemblages indicate that these types of alteration were associated with REE fractionation. These data show that $\mathrm{REE}, \mathrm{Nb}$ and $\mathrm{Zr}$ were mobile in the late-magmatic hydrothermal environment. Initial $\mathrm{Nd}$ isotope ratios of fresh eudialyte and catapleiite- and gittinsite-pseudomorphs are indistinguishable at the $95 \%$ confidence limit, consistent with a magmatic origin for the associated fluids. By contrast, we infer that the positive $\varepsilon \mathrm{Nd}(\mathrm{t})(+0.5)$ of the zircon-bearing pseudomorphs reflects significant isotopic disturbance by the alteration fluids, consistent with an external fluid source. The narrow range of weakly negative $\varepsilon N d(t)$ values $(-0.8$ to -1.1$)$ found for eudialyte, pseudomorphs and three REE-phases supports the model of closed-system fractionation of a previously contaminated mantle melt for the agpaitic portion of the Ilímaussaq complex. While the present work shows that HFSE are removed from the original crystal volume, we infer that these elements were largely retained within the kakortokite. Targeted processing of particular secondary mineral groups could significantly enhance metal recovery from the alteration assemblage and potentially simplify the REE separation process. Coupled mineralogical and metallurgical studies should evaluate the physical and chemical properties of the alteration assemblages to maximise metal recovery from eudialyte-hosted deposits.

Supplementary Materials: The following are available online at http:/ /www.mdpi.com/2075-163X/9/7/422/s1, Figure S1: photoscans of the sample slabs, Figure S2: photoscan of sample 109202 separated into four mineral classes, Figure S3: trace element budgets of matrix minerals, Figure S4: Results of matrix dilution modelling, Table S1: simplified sample modal compositions determined by image analysis, Table S2: TIMS Nd standard data, Table S3: TIMS Sm standard data, Table S4: $t$-test comparing initial Nd ratios of eudialyte and pseudomorphs.

Author Contributions: Conceptualisation, M.A.J.v.d.V., A.M.B. and A.A.F.; Funding acquisition, G.R.D.; Investigation, M.A.J.v.d.V. and A.M.B.; Methodology, M.A.J.v.d.V., A.M.B. and A.A.F.; Project administration, M.A.J.v.d.V., A.M.B. and A.A.F.; Resources, A.M.B., G.R.D., E.J.H. and A.A.F.; Supervision, A.M.B., G.R.D. and A.A.F.; Validation, M.A.J.v.d.V., A.M.B., G.R.D., E.J.H. and A.A.F.; Visualisation, M.A.J.v.d.V.; Writing-original draft, M.A.J.v.d.V., A.M.B. and A.A.F.; Writing—review and editing, M.A.J.v.d.V., A.M.B., G.R.D., E.J.H. and A.A.F.

Funding: M.A.J.v.d.V., A.M.B. and A.A.F. were funded by the NERC-funded SOS RARE consortium, Grant No. NE/M010856/1.

Acknowledgments: The authors thank the Geological Survey of Denmark and Greenland, Henning Bohse, John Bailey and Nicky Horsburgh for permission to use their samples. The authors also thank Richard Smeets, Kirsten van Zuilen and Paolo D'Imporanzo for assistance with sample preparation and analysis. The authors thank four anonymous reviewers for reviewing an earlier version of this manuscript. Academic Editor Stefano Salvi and Assistant Editors of Minerals are thanked for editorial handing of the manuscript.

Conflicts of Interest: The authors declare no conflict of interest.

\section{Appendix A. Full Sample Descriptions}

\section{Appendix A.1. 109202 (Catapleiite-Type)}

Sample 109202 originates from layer 0R (red kakortokite, unit 0). Euhedral eudialyte and hydrothermally altered eudialyte $(\leq 1 \mathrm{~mm})$ comprise about $70 \%$ of the mode, the interstitial space filled with nepheline, feldspar (combined about 25\%) and arfvedsonite (about 5\%). Preserved eudialyte and their pseudomorphs both exhibit the typical octagonal morphology. Approximately half of the eudialyte present in this sample has undergone hydrothermal alteration and secondary catapleiite, 
aegirine and analcime are readily identified under the petrographic microscope. Alteration ranges from complete to partial and generally progresses inwards from the margins and cracks within the grain.

\section{Appendix A.2. 540286 (Catapleiite-Type)}

Sample 540286 derives from layer 13R (red kakortokite, unit 13) and constitutes a mineralogy of euhedral eudialyte and hydrothermally altered eudialyte ( $\leq 1 \mathrm{~mm}$, about $30 \%)$, equant nepheline ( $\leq 1 \mathrm{~mm}$, about $15 \%$ ), elongate and slender laths of feldspar ( $\leq 7 \mathrm{~mm}$ long, about $30 \%$ ) and subhedral arfvedsonite ( $\leq 3 \mathrm{~mm}$, about $25 \%$ ). Eudialyte alteration is pervasive with about $75 \%$ of eudialyte crystals affected. Alteration, where present, is advanced such that crystals are either unaffected or completely replaced. Elongate laths of catapleiite, green-brownish aegirine and interstitial analcime are readily identified in the secondary assemblages using the petrographic microscope.

\section{Appendix A.3. 540269 (Catapleiite-Type)}

Sample 540269 was collected from layer TLK-A red (red transitional kakortokite, unit A), just below a pegmatite horizon. The sample displays a saccharoidal texture comprising predominantly fine-grained euhedral eudialyte ( $\leq 0.5 \mathrm{~mm}$, about $40 \%$, most of which is altered), fine prismatic aegirine ( $\leq 0.2 \mathrm{~mm}$, about $20 \%$ ), equant nepheline (about $15 \%)$, small laths of alkali feldspar $(\leq 0.2$ $\mathrm{mm}$, about $10 \%)$, few larger crystals of sodalite $(\leq 0.8 \mathrm{~mm}$, about $10 \%)$ and interstitial analcime (about $5 \%$ ). The eudialyte pseudomorph assemblage is fine-grained and appears opaque in hand specimens, but aegirine, catapleiite and analcime can be identified under the petrographic microscope. Alteration of eudialyte in this specimen affected about $70 \%$ of the crystals. A spatial correlation is observed in the polished slab with unaltered eudialyte clustered in a band of about $1.5 \mathrm{~cm}$ wide and $3 \mathrm{~cm}$ long and altered eudialyte occupying the surrounding volume.

\section{Appendix A.4. 109211 (Gittinsite-Type)}

Sample 109211 was sampled from layer 3B (black kakortokite, unit 3). Subhedral arfvedsonite $(\leq 5 \mathrm{~mm}$ ) dominates the mineralogy of this specimen and occupies about $40 \%$ of the rock volume. The remaining mineralogy comprises subhedral nepheline (about 20\%), alkali feldspar (about 20\%), interstitial sodalite (about $10 \%)$, and euhedral or interstitial eudialyte $(<10 \%)$. Eudialyte alteration is pervasive and clear vitreous crystals are rare. The alteration assemblage replacing eudialyte is generally too fine for identification optically, but gittinsite was identified as the secondary zirconosilicate in this specimen by Borst et al. [27]. Other minerals occurring in the gittinsite-pseudomorph assemblages include annite, fergusonite-(Y), allanite-(Ce) and secondary rims of Sr-rich eudialyte [27].

\section{Appendix A.5. EJH/12/091 (Zircon-Type)}

Sample EJH/12/091 was obtained from the "hybrid-type" kakortokite and is a coarse-grained (crystal sizes up to $4 \mathrm{~mm}$ ) rock. It is principally composed of alkali feldspar laths ( $\geq 5 \mathrm{~mm}$ long, about $60 \%$ ), with subhedral to anhedral arfvedsonite ( $\geq 3 \mathrm{~mm}$, about $20 \%$ ), subhedral to euhedral nepheline ( $\geq 1 \mathrm{~mm}$, about $10 \%$ ) and pseudomorphed eudialyte (about $10 \%)$. All of the eudialyte in this sample has been altered to a fine-grained assemblage ( $\geq 1 \mathrm{~mm})$, from which zircon, aegirine, alkali feldspar and catapleiite can be identified under the petrographic microscope.

\section{References}

1. USGS. Rare Earths: Mineral Commodity Summaries 2018; Technical Report; United States Geological Survey: Reston, VA, USA, 2019.

2. Hatch, G.P. Dynamics in the Global Market for Rare Earths. Elements 2012, 8, 341-346. [CrossRef]

3. Barakos, G.; Gutzmer, J.; Mischo, H. Strategic evaluations and mining process optimization towards a strong global REE supply chain. J. Sustain. Min. 2016, 15, 26-35. [CrossRef] 
4. Jordens, A.; Cheng, Y.P.; Waters, K.E. A review of the beneficiation of rare earth element bearing minerals. Miner. Eng. 2013, 41, 97-114. [CrossRef]

5. Zepf, V. The Way to the Chinese Predominance: A Key for Understanding the REE Issue. In Rare Earth Elements; Springer: Berlin/Heidelberg, Germany, 2011; Chapter 3; pp. 41-49.

6. Brumme, A. Wind Energy Deployment and the Relevance of Rare Earths. In Wind Energy Deployment and the Relevance of Rare Earths; Springer Fachmedien Wiesbaden: Wiesbaden, Germany, 2014; Chapter 3; pp. 17-48.

7. Haque, N.; Hughes, A.; Lim, S.; Vernon, C. Rare Earth Elements: Overview of Mining, Mineralogy, Uses, Sustainability and Environmental Impact. Resources 2014, 3, 614-635. [CrossRef]

8. Van Gosen, B.S.; Verplanck, P.L.; Seal, R.R., II; Long, K.R.; Gambogi, J. Rare-Earth Elements, Chap. O of Critical Mineral Resources of the United States-Economic and Environmental Geology and Prospects for Future Supply. In U.S. Geological Survey Professional Paper 1802; Schulz, K.J., DeYoung, J.H., Seal, R.R., II, Bradley, D.C., Eds.; USGS: Reston, VA, USA, 2017; Chapter O.; pp. 1-31. [CrossRef]

9. Adamas Intelligence. Rare Earth Market Outlook: Supply, Demand, and Pricing from 2016 through 2025: Executive Summary; Technical Report; Adamas Intelligence: Amsterdam, The Netherlands, 2016.

10. Sørensen, H. Agpaitic nepheline syenites: A potential source of rare elements. Appl. Geochem. 1992, 7, 417-427. [CrossRef]

11. Bailey, J.; Gwozdz, R.; Rose-Hansen, J. Geochemical overview of the Ilímaussaq alkaline complex, South Greenland. Geol. Greenl. Surv. Bull. 2001, 190, 35-53.

12. TANBREEZ. Tanbreez Ltd., Company Website. Available online: http://tanbreez.com/ (accessed on 30 May 2019) .

13. Bohse, H.; Brooks, C.; Kunzendorf, H. Field Observations on the Kakortokites of the Ilimaussaq Intrusion, South Greenland, Including Mapping and Analyses by Portable X-ray Fluorescence Equipment for Zirconium and Niobium; Technical Report; University of Copenhagen: Copenhagen, Denmark, 1971.

14. Le Maitre, R.W.; Streckeisen, A.; Zanettin, B.; Le Bas, M.J.; Bonin, B.; Bateman, P.; Bellieni, G.; Dudek, A.; Efremova, S.; Keller, J.; et al. Igneous Rocks: A Classification and Glossary of Terms; Cambridge University Press: Cambridge, UK, 2003; p. 252. [CrossRef]

15. Johnsen, O.; Ferraris, G.; Gault, R.A.; Grice, J.D.; Kampf, A.R.; Pekov, I.V. The nomenclature of eudialyte-group minerals. Can. Mineral. 2003, 41, 785-794. [CrossRef]

16. Borst, A.; Friis, H.; Nielsen, T.F.D.; Waight, T.E. Bulk and mush melt evolution in agpaitic intrusions: Insights from compositional zoning in eudialyte, Ilímaussaq complex, South Greenland. J. Petrol. 2018, 59, 589-612. [CrossRef]

17. Chakhmouradian, A.R.; Wall, F. Rare earth elements: Minerals, mines, magnets (and more). Elements 2012, 8, 333-340. [CrossRef]

18. Schilling, J.; Wu, F.; McCammon, C.; Wenzel, T.; Marks, M.A.; Pfaff, K.; Jacob, D.E.; Markl, G. The compositional variability of eudialyte-group minerals. Mineral. Mag. 2011, 75, 87-115. [CrossRef]

19. Stark, T.; Silin, I.; Wotruba, H. Mineral Processing of Eudialyte Ore from Norra Kärr. J. Sustain. Metall. 2017, 3, 32-38. [CrossRef]

20. Davris, P.; Stopic, S.; Balomenos, E.; Panias, D.; Paspaliaris, I.; Friedrich, B. Leaching of rare earth elements from eudialyte concentrate by suppressing silica gel formation. Miner. Eng. 2017, 108, 115-122. [CrossRef]

21. Voßenkaul, D.; Birich, A.; Müller, N.; Stoltz, N.; Friedrich, B. Hydrometallurgical Processing of Eudialyte Bearing Concentrates to Recover Rare Earth Elements Via Low-Temperature Dry Digestion to Prevent the Silica Gel Formation. J. Sustain. Metall. 2017, 3, 79-89. [CrossRef]

22. Coulson, I.M. Post-magmatic alteration in eudialyte from the North Qôroq centre, South Greenland. Mineral. Mag. 1997, 61, 99-109. [CrossRef]

23. Mitchell, R.H.; Liferovich, R.P. Subsolidus deuteric/hydrothermal alteration of eudialyte in lujavrite from the Pilansberg alkaline complex, South Africa. Lithos 2006, 91, 352-372. [CrossRef]

24. Mitchell, R.H.; Chakrabarty, A. Paragenesis and decomposition assemblage of a Mn-rich eudialyte from the Sushina peralkaline nepheline syenite gneiss, Paschim Banga, India. Lithos 2012, 152, 218-226. [CrossRef]

25. Estrade, G.; Salvi, S.; Béziat, D. Crystallization and destabilization of eudialyte-group minerals in peralkaline granite and pegmatite: A case study from the Ambohimirahavavy complex, Madagascar. Mineral. Mag. 2018, 82, 375-399. [CrossRef]

26. Möller, V.; Williams-Jones, A. Magmatic and hydrothermal controls on the mineralogy of the basal zone, Nechalacho REE-Nb-Zr deposit, Canada. Econ. Geol. 2017, 112, 1823-1856. [CrossRef] 
27. Borst, A.; Friis, H.; Andersen, T.; Nielsen, T.F.D.; Waight, T.E.; Smit, M.A. Zirconosilicates in the kakortokites of the Ilímaussaq complex, South Greenland: Implications for fluid evolution and high-field-strength and rare-earth element mineralization in agpaitic systems. Mineral. Mag. 2016, 80, 5-30. [CrossRef]

28. Marks, M.A.; Markl, G. A global review on agpaitic rocks. Earth-Sci. Rev. 2017, 173, 229-258. [CrossRef]

29. Karup-Møller, S.; Rose-Hansen, J.; Sørensen, H. Eudialyte decomposition minerals with new hitherto undescribed phases from the Ilímaussaq complex, South Greenland. Bull. Geol. Soc. Den. 2010, 58, 75-88.

30. Karup-Møller, S.; Rose-Hansen, J. New data on eudialyte decomposition minerals from kakortokites and associated pegmatites of the Ilímaussaq complex, South Greenland. Bull. Geol. Soc. Den. 2013, 61, 47-70.

31. Ussing, N. Geology of the Country Around Julianehaab, Greenland; Bianco Luno: Copenhagen, Denmark, 1911; p. 376, Number 1.

32. Hunt, E.J. Magma Chamber Dynamics in the Peralkaline Magmas of the Kakortokite Series, South Greenland. Ph.D. Thesis, University of St Andrews, St Andrews, UK, 2015. [CrossRef]

33. Krumrei, T.V.; Villa, I.M.; Marks, M.A.; Markl, G. A 40Ar/39Ar and U/Pb isotopic study of the Ilímaussaq complex, South Greenland: Implications for the 40K decay constant and for the duration of magmatic activity in a peralkaline complex. Chem. Geol. 2006, 227, 258-273. [CrossRef]

34. Upton, B. Tectono-magmatic evolution of the younger Gardar southern rift, South Greenland. Geol. Surv. Den. Greenl. Bull. 2013, 2013, 1-128. [CrossRef]

35. Upton, B.; Emeleus, C.H.; Heaman, L.M.; Goodenough, K.; Finch, A.A. Magmatism of the mid-Proterozoic Gardar Province, South Greenland: Chronology, petrogenesis and geological setting. Lithos 2003, 68, 43-65. [CrossRef]

36. Konnerup-Madsen, J.; Rose-Hansen, J. Composition and significance of fluid inclusions in the Ilímaussaq peralkaline granite, South Greenland. Bull. De Minéralogie 1984, 107, 317-326. [CrossRef]

37. Larsen, L.M.; Sorensen, H. The Ilímaussaq intrusion-progressive crystallization and formation of layering in an agpaitic magma. Geol. Soc. Lond. Spec. Publ. 1987, 30, 473-488. [CrossRef]

38. Garde, A.A.; Hamilton, M.A.; Chadwick, B.; Grocott, J.; McCaffrey, K.J. The Ketilidian orogen of South Greenland: Geochronology, tectonics, magmatism, and fore-arc accretion during Palaeoproterozoic oblique convergence. Can. J. Earth Sci. 2002, 39, 765-793. [CrossRef]

39. Sørensen, H.; Bohse, H.; Bailey, J. The origin and mode of emplacement of lujavrites in the Ilímaussaq alkaline complex, South Greenland. Lithos 2006, 91, 286-300. [CrossRef]

40. Marks, M.A. Layered intrusions. In Layered Intrusions; Charlier, B., Namur, O., Rais, L., Tegner, C., Eds.; Springer: Berlin, Germany, 2015; Chapter 14; pp. 649-691. [CrossRef]

41. Ratschbacher, B.C.; Marks, M.A.; Bons, P.D.; Wenzel, T.; Markl, G. Emplacement and geochemical evolution of highly evolved syenites investigated by a combined structural and geochemical field study: The lujavrites of the Ilímaussaq complex, SW Greenland. Lithos 2015, 231, 62-76. [CrossRef]

42. Borst, A.; Waight, T.E.; Finch, A.A.; Storey, M.; Roux, P.J. Dating agpaitic rocks: A multi-system (U/Pb, $\mathrm{Sm} / \mathrm{Nd}, \mathrm{Rb} / \mathrm{Sr}$ and $40 \mathrm{Ar} / 39 \mathrm{Ar}$ ) isotopic study of layered nepheline syenites from the Ilímaussaq complex, Greenland. Lithos 2019, 324-325, 74-88. [CrossRef]

43. Charlier, B.; Ginibre, C.; Morgan, D.; Nowell, G.M.; Pearson, D.G.; Davidson, J.P.; Ottley, C.J. Methods for the microsampling and high-precision analysis of strontium and rubidium isotopes at single crystal scale for petrological and geochronological applications. Chem. Geol. 2006, 232, 114-133. [CrossRef]

44. Vilalva, F.C.; Vlach, S.R.; Simonetti, A. Nacareniobsite-(Ce) and britholite-(Ce) in peralkaline granites from the Morro Redondo Complex, Graciosa Province, Southern Brazil: Occurrence and compositional data. Can. Mineral. 2013, 51, 313-332. [CrossRef]

45. Chakrabarty, A.; Mitchell, R.H.; Ren, M.; Sen, A.K.; Pruseth, K.L. Rinkite, cerianite-(Ce), and hingganite-(Ce) in syenite gneisses from the Sushina Hill Complex, India: Occurrence, compositional data and petrogenetic significance. Mineral. Mag. 2013, 77, 3137-3153. [CrossRef]

46. Pekov, I.V.; Ekimenkova, I.A. Two new rare-earth-rich mineral associations in the Ilímaussaq alkaline complex, South Greenland. Geol. Greenl. Surv. Bull. 2001, 190, 143-144.

47. Frei, D.; Liebscher, A.; Franz, G.; Berlin, D.; Dulski, P. Trace Element Geochemistry. Rev. Mineral. Geochem. 2004, 56, 553-605. [CrossRef]

48. Tanaka, T.; Togashi, S.; Kamioka, H.; Amakawa, H.; Kagami, H.; Hamamoto, T.; Yuhara, M.; Orihashi, Y.; Yoneda, S.; Shimizu, H.; et al. JNdi-1: A neodymium isotopic reference in consistency with LaJolla neodymium. Chem. Geol. 2000, 168, 279-281. [CrossRef] 
49. McDonough, W.F.; Sun, S.S. The composition of the Earth. Chem. Geol. 1995, 120, 223-253. [CrossRef]

50. Pfaff, K.; Krumrei, T.V.; Marks, M.A.; Wenzel, T.; Rudolf, T.; Markl, G. Chemical and physical evolution of the 'lower layered sequence' from the nepheline syenitic Ilímaussaq intrusion, South Greenland: Implications for the origin of magmatic layering in peralkaline felsic liquids. Lithos 2008, 106, 280-296. [CrossRef]

51. Marks, M.A.; Vennemann, T.; Siebel, W.; Markl, G. Nd-, O-, and H-isotopic evidence for complex, closed-system fluid evolution of the peralkaline Ilímaussaq intrusion, south Greenland. Geochim. Cosmochim. Acta 2004, 68, 3379-3395. [CrossRef]

52. Lugmair, G.W. Sm-Nd ages: A new dating method. Meteoritics 1974, 9, 369.

53. Goldstein, S.; O'Nions, S.; Hamilton, P. A Sm-Nd isotopic study of atmospheric dusts and particulates from major river systems. Earth Planet. Sci. Lett. 1984, 70, 221-236. [CrossRef]

54. Jacobsen, S.B.; Wasserburg, G.J. Sm-Nd isotopic evolution of chondrites. Earth Planet. Sci. Lett. 1980, 50, 139-155. [CrossRef]

55. Friis, H. Primary and secondary mineralogy of the Ilímaussaq alkaline complex, South Greenland. In Proceedings of the Symposium on Strategic and Critical Materials Proceedings, Victoria, BC, Canada, 13-14 November 2015; Simandl, G., Neetz, M., Eds.; British Columbia Ministry of Energy and Mines: Victoria, BC, Canada, 2015; Volume 3, pp. 83-89.

56. Salvi, S.; Fontan, F.; Monchoux, P.; Williams-Jones, A.; Moine, B. Hydrothermal Mobilization of High Field Strength Elements in Alkaline Igneous Systems: Evidence from the Tamazeght Complex (Morocco). Econ. Geol. 2000, 95, 559-579. [CrossRef]

57. Paslick, C.R.; Halliday, A.N.; Davies, G.R.; Mezger, K.; Upton, B. Timing of Proterozoic magmatism in the Gardar Province, southern Greenland. Geol. Soc. Am. Bull. 1993, 105, 272-278. [CrossRef]

58. Marks, M.A.; Markl, G. Ilímaussaq “en miniature": Closed-system fractionation in an agpaitic dyke rock from the Gardar Province, South Greenland (contribution to the mineralogy of Ilímaussaq no.117). Mineral. Mag. 2003, 67, 893-919. [CrossRef]

59. Chao, E.C.; Back, J.M.; Minkin, J.A.; Yinchen, R. Host-rock controlled epigenetic, hydrothermal metasomatic origin of the Bayan Obo REEFe-Nb ore deposit, Inner Mongolia, P.R.C. Appl. Geochem. 1992, 7, 443-458. [CrossRef]

60. Boily, M.; Williams-Jones, A. The role of magmatic and hydrothermal processes in the chemical evolution of the Strange Lake plutonic complex, Québec-Labrador. Contrib. Mineral. Petrol. 1994, 118, 33-47. [CrossRef]

61. Salvi, S.; Williams-Jones, A. The role of hydrothermal processes in concentrating high-field strength elements in the Strange Lake peralkaline complex, northeastern Canada. Geochim. Cosmochim. Acta 1996, 60, 1917-1932. [CrossRef]

62. Kynicky, J.; Chakhmouradian, A.R.; Xu, C.; Krmicek, L.; Galiova, M. Distribution and evolution of zirconium mineralization in peralkaline granites and associated pegmatites of the Khan Bogd complex, southern Mongolia. Can. Mineral. 2011, 49, 947-965. [CrossRef]

63. Sheard, E.R.; Williams-Jones, A.; Heiligmann, M.; Pederson, C.; Trueman, D.L. Controls on the concentration of zirconium, niobium, and the rare earth elements in the Thor Lake rare metal deposit, Northwest Territories, Canada. Econ. Geol. 2012, 107, 81-104. [CrossRef]

64. Gysi, A.P.; Williams-Jones, A. Hydrothermal mobilization of pegmatite-hosted REE and Zr at Strange Lake, Canada: A reaction path model. Geochim. Cosmochim. Acta 2013, 122, 324-352. [CrossRef]

65. Estrade, G.; Salvi, S.; Béziat, D.; Williams-Jones, A. The origin of skarn-hosted rare-metal mineralization in the Ambohimirahavavy alkaline complex, Madagascar. Econ. Geol. 2015, 110, 1485-1513. [CrossRef]

66. Hutchison, W.; Babiel, R.; Finch, A.; Marks, M.; Markl, G.; Boyce, A.; Stueeken, E.; Friis, H.; Borst, A.; Horsburgh, N. Sulphur isotopes of alkaline magmas unlock long-term records of crustal recycling on Earth. Nat. Commun. 2019, under review.

67. Pearson, R.G. Hard and Soft Acids and Bases. J. Am. Chem. Soc. 1963, 85, 3533-3539. [CrossRef]

68. Migdisov, A.A.; Williams-Jones, A.; Wagner, T. An experimental study of the solubility and speciation of the Rare Earth Elements (III) in fluoride- and chloride-bearing aqueous solutions at temperatures up to $300{ }^{\circ} \mathrm{C}$. Geochim. Cosmochim. Acta 2009, 73, 7087-7109. [CrossRef]

69. Migdisov, A.A.; Williams-Jones, A. Hydrothermal transport and deposition of the rare earth elements by fluorine-bearing aqueous liquids. Miner. Depos. 2014, 49, 987-997. [CrossRef] 
70. Migdisov, A.A.; Williams-Jones, A.; Brugger, J.; Caporuscio, F.A. Hydrothermal transport, deposition, and fractionation of the REE: Experimental data and thermodynamic calculations. Chem. Geol. 2016, 439, 13-42. [CrossRef]

71. Migdisov, A.A.; Williams-Jones, A.; van Hinsberg, V.; Salvi, S. An experimental study of the solubility of baddeleyite $(\mathrm{ZrO} 2)$ in fluoride-bearing solutions at elevated temperature. Geochim. Cosmochim. Acta 2011, 75, 7426-7434. [CrossRef]

72. Timofeev, A.; Migdisov, A.A.; Williams-Jones, A. An experimental study of the solubility and speciation of tantalum in fluoride-bearing aqueous solutions at elevated temperature. Geochim. Cosmochim. Acta 2017, 197, 294-304. [CrossRef]

73. Markl, G.; Baumgartner, L. pH changes in peralkaline late-magmatic fluids. Contrib. Mineral. Petrol. 2002, 144, 331-346. [CrossRef]

74. Stevenson, R.; Upton, B.; Steenfelt, A. Crust-mantle interaction in the evolution of the Ilímaussaq Complex, South Greenland: Nd isotopic studies. Lithos 1997, 40, 189-202. [CrossRef]

75. Goodenough, K.; Upton, B.; Ellam, R. Long-term memory of subduction processes in the lithospheric mantle: Evidence from the geochemistry of basic dykes in the Gardar Province of South Greenland. J. Geol. Soc. 2002, 159, 705-714. [CrossRef]

76. Halama, R.; Wenzel, T.; Upton, B.; Siebel, W.; Markl, G. A geochemical and Sr-Nd-O isotopic study of the Proterozoic Eriksfjord Basalts, Gardar Province, South Greenland: Reconstruction of an OIB signature in crustally contaminated rift-related basalts. Mineral. Mag. 2003, 67, 831-853. [CrossRef]

77. Barnes, G. (Tanbreez Ltd.). Personal Communication. 2018.

(C) 2019 by the authors. Licensee MDPI, Basel, Switzerland. This article is an open access article distributed under the terms and conditions of the Creative Commons Attribution (CC BY) license (http:/ / creativecommons.org/licenses/by/4.0/). 